\title{
Tracking of Range and Azimuth for Continuous Imaging of Marine Target in Monopulse ISAR with Wideband Echoes
}

\author{
Junhao Xie, Wei Zhou, Gongjian Zhou, Yeshu Yuan, and Shaobin Li \\ Department of Electronic Engineering, Harbin Institute of Technology, Harbin 150001, China \\ Correspondence should be addressed to Junhao Xie; xj@hit.edu.cn and Gongjian Zhou; zhougj@hit.edu.cn
}

Received 29 October 2015; Revised 3 March 2016; Accepted 22 March 2016

Academic Editor: Wei Liu

Copyright (C) 2016 Junhao Xie et al. This is an open access article distributed under the Creative Commons Attribution License, which permits unrestricted use, distribution, and reproduction in any medium, provided the original work is properly cited.

\begin{abstract}
Real-time tracking of maneuvering targets is the prerequisite for continuous imaging of moving targets in inverse synthetic aperture radar (ISAR). In this paper, the range and azimuth tracking (RAT) method with wideband radar echoes is first presented for a mechanical scanning monopulse ISAR, which is regarded as the simplest phased array unit due to the two antenna feeds. To relieve the estimation fluctuation and poor robustness of the RAT method with a single snapshot, a modified range and azimuth tracking approach based on centroid algorithm (RATCA) with forgotten factor and multiple echoes is then proposed. The performances of different forgotten factors are investigated. Both theoretical analysis and experimental results demonstrate that RATCA is superior to RAT method. Particularly, when target echo is missing occasionally, RAT method fails while RATCA still keeps good performance. The potential of continuous imaging with shipborne ISAR is verified by experimental results. With minor modification, the method proposed in this paper can be potentially applied in the phased array radar.
\end{abstract}

\section{Introduction}

Inverse synthetic aperture radar (ISAR) has been widely employed to obtain the high-resolution image of the target for coastal surveillance and target recognition [1-3]. In recent decades, many imaging approaches such as range-instantaneous-Doppler algorithm [4-6], time-frequency processing method [7-9], and compressive sensing algorithm [10-12] were proposed. For all these advanced imaging algorithms, the image quality heavily relies on the correctness of recorded data.

In conventional ISAR, the imaging processing consists of two successive stages: (1) detect and estimate the position of target by narrow bandwidth echoes and (2) use the wideband echo to obtain the ISAR image of the prelocated target. In this kind of system, to redetect the target after imaging is necessary because of the range shift and angular change in the imaging duration, which is typically several seconds for marine target. Due to the redetection procedure, the time difference between two consecutive imaging processes is almost the same as the antenna scan period, which is too long for coastal ISAR system, and the ability of surveillance is seriously limited. Therefore, in this paper, we propose a wideband target tracking technique which allows ISAR system continuous imaging without the redetection procedure. Thus, the ISAR images of the same threatening target from different views could be continuously obtained. This is of great importance for target recognition. Because the redetection is canceled, range and azimuth tracking simultaneously is required to capture the target by adjusting the radar line of sight (LOS) and receiver sampling gate adaptively.

Range tracking is to record only the effective target data in each echo according to the estimated time delay of the target. This decreases the quantity of recorded data largely and is beneficial to the subsequent digital signal processing. A conventional method is to track the prominent scatterers on the target [1], but it is not always effective due to the scintillation or shadowing effect. Delisle and $\mathrm{Wu}$ [13] propose an average envelope correlation algorithm with Kalman filter, which has good tracking performance but may be computationally prohibitive. To track the target centroid is an alternative way, which is simple in designing and effective in processing. By using the azimuth tracking, a moving target could be illuminated permanently by the antenna, in which the radar 
LOS is modified in real time by the estimation of target azimuth. A typical approach is to estimate the angular motion by comparing the phase difference of different scatterers [14], but the reliable reference scatterers are hard to select in practice due to the disturbances from the noise and clutter. A differential phase shift method was presented by using the phase difference [15], which is introduced by the differential time delay between two receivers after range tracking and range bin alignment, and thus the angle accuracy depends on the range measurement. Monopulse method [16], in which the core issue is to estimate the instantaneous angle-error [17], is widely used in modern radar for target tracking. In the practical ISAR, two independent channels for azimuth coordinate are generated to form a monopulse system. Target wideband signals received by sum and difference channels, which are formed by the magic T module, are utilized to sense the target angle-error for adjusting the radar LOS adaptively. The latest angle-error of the target could be estimated from a single pulse with the well-known sum-difference amplitudecomparison monopulse method.

In this paper, a novel instantaneous range and azimuth tracking (RAT) algorithm based on a single wideband echo is proposed for continuous imaging of marine target. To improve the tracking performance and robustness of RAT method under some extreme conditions such as target echo missing, a modified range and azimuth tracking approach based on centroid algorithm (RATCA) is further proposed. Both RAT and RATCA are real-time tracking methods; however, RATCA extends the RAT method by using multiple wideband echoes and employing forgotten factors. These proposed methods are verified by the experiments of shipborne ISAR system. Note that the methods proposed in this paper can be easily extended to the case of phase array systems with numerous transmit/receive (T/R) modules.

The rest of this paper is organized as follows. The RAT algorithm for a single snapshot is presented in Section 2 . The RATCA algorithm for multiple snapshots is proposed in Section 3 to improve the range and azimuth tracking performance. Section 4 presents the experimental results and theoretical analysis, followed by conclusion in Section 5 .

\section{Continuous Imaging and RAT Algorithm}

2.1. Descriptions of Continuous Imaging in ISAR. Modern radar system needs not only to estimate the target tracks but also to obtain ISAR images of targets for data fusion and recognition. As mentioned before, to detect and to obtain the image of the target, respectively, by short time division multiplexing is a conventional process in traditional radar system. Detecting and imaging are two main operation modes of the practical ISAR. In detecting mode, radar transmits short linear frequency modulation (LFM) pulse with narrow bandwidth to sense the presence of targets and estimate their ranges and azimuths as a priori knowledge for imaging. When radar is operated in imaging mode, the bandwidth of the transmitted LFM pulse is typically several hundreds of megahertz to achieve the high range resolution.
The ISAR image contains many details of targets and now plays an important role in marine surveillance. From the high range resolution image, more information about the target of interest, such as attitude, size, and type, can be inferred. To monitor and obtain the images of an unknown marine target continuously is of great significance in coastal surveillance. However, the redetection procedure is required in conventional radar system and the continuous imaging is unable to be implemented. By considering radar scan period, redetection may take a long time in searching the target of interest between successive imaging processes; hence, the ability to monitor some threatening target is limited. Therefore, target continuous imaging based on wideband echoes without any redetection is preferred for some threatening targets.

The most challenging part in continuous imaging is target tracking during the imaging processing. In high range resolution radar, only the effective target data is recorded for range tracking by a setup sampling gate after stretch processing, which is widely employed to reduce the effective baseband signal bandwidth substantially and is an advantage of data sampling and following digital signal processing. On the other hand, imaging target needs to be illuminated by the antenna to obtain the target returns. Ideally, target should be located in the center of imaging range scene and the main beam of antenna as shown in Figure 1. If the system could not adjust the sampling gate and antenna LOS simultaneously for maneuvering target, the target may move away from the original imaging range scene or main beam of antenna during the imaging processing. Thus, the echo will be lost and the ISAR imaging will fail.

2.2. Principle of RAT Method. For tracking and imaging simultaneously, the RAT method is proposed in this section. RAT method is a signal processing method based on high range resolution profile (HRRP) of the target consisting of numerous scatterers, which are uniformly distributed in range, with different amplitudes and phases. RAT method consists of range tracking and azimuth tracking, as shown in Figure 2, where the sampling gate and LOS of antenna will be modified adaptively with the radial and angular motion of the target.

2.2.1. Range Tracking. The main task of range tracking is to estimate the range deviation from the center of the imaging scene. The first local oscillator (LO) delay is then modified according to the estimated range deviation, also called rangeerror, to record only the effective target data for further processing.

In high-resolution radar, the major scattering features could be projected into a range profile, which consists of numerous scatterers in each position with different amplitudes and phases. Stretch processing is a technique for wideband LFM pulse compression, allowing the range of the target in time-domain to be represented by a corresponding frequency. A stretch processor allows the effective baseband signal of the target to be significantly reduced. The range profile digital sequence, which contains details of target 


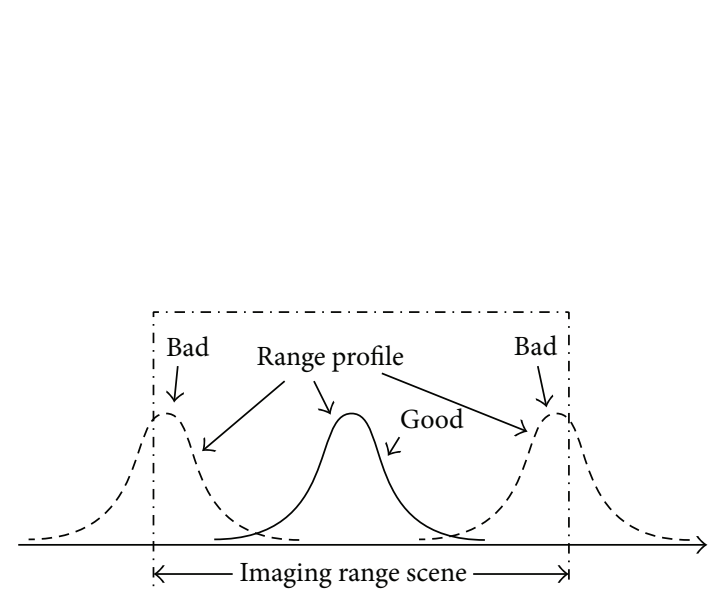

(a)

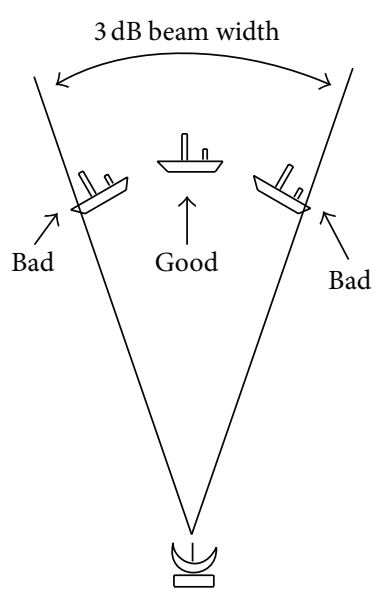

(b)

FIGURE 1: Different positions of the target. (a) Different positions of the target in the imaging range scene. (b) Different positions of the target in the radar antenna beam.

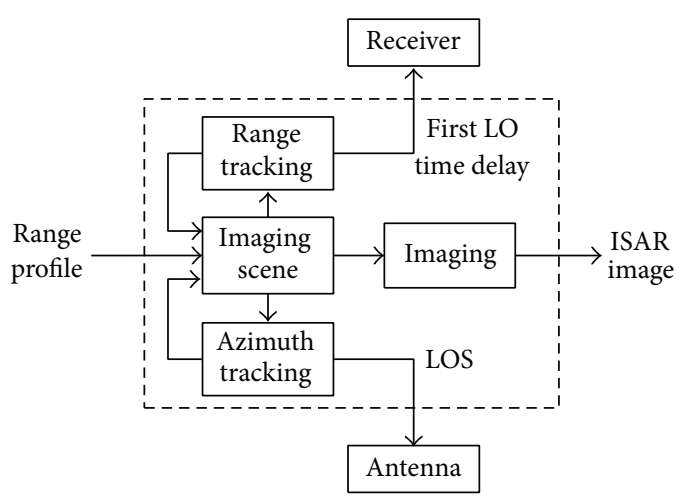

(a)

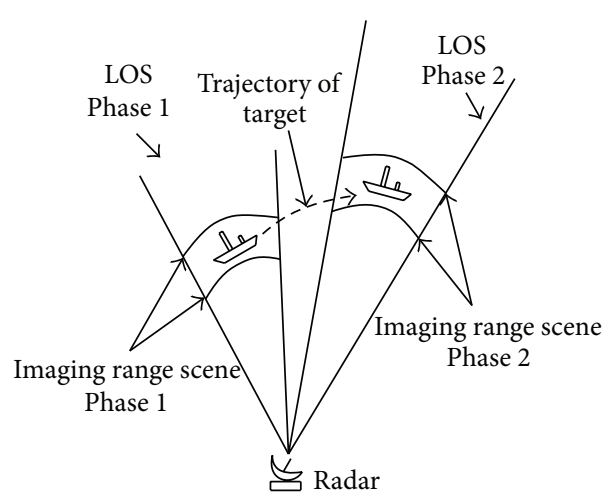

(b)

Figure 2: Principles of RAT method. (a) Basic block diagram of imaging processing with range and azimuth tracking. (b) Tracking of marine target in ISAR imaging.

such as amplitude, phase, and location in range, could be obtained via fast Fourier transform (FFT) of the baseband data after stretch processing. A slide window with given length smoothens the random fluctuation of the range profile to obtain the contour of the target, as illustrated in Figure 3(a). Thus, the target could be reasonably extracted from the smoothed sequence with a given threshold, and the rangeerror $\Delta R$ could be estimated as in Figure 3 .

Assume that there are $N$ prominent scatterers beyond the fixed threshold in target range profile that are used to estimate the target range. Denote by $R_{L}$ the width of imaging scene scale and by $L_{\mathrm{FFT}}$ the total range bin number in the range profile digital sequence after FFT; the length of each range bin is calculated by

$$
\begin{aligned}
R_{\mathrm{bin}} & =\frac{R_{L}}{L_{\mathrm{FFT}}}, \\
R_{L} & =\frac{c T_{r} f_{s}}{2 B},
\end{aligned}
$$

where $c$ is the speed of light, $T_{r}$ is pulse repetition interval (PRI), $f_{s}$ is the sample rate, and $B$ is the transmit bandwidth. Suppose that the range bin order of each selected scatterer is denoted by $J_{i}, i \in[1, N]$; the average range estimate could be expressed as

$$
R_{\mathrm{av}}=R_{\mathrm{bin}} \cdot \frac{\sum_{i=1}^{N} J_{i}}{N} .
$$

The range-error, which is the key quantity for adjusting the first LO delay in data sampling, can be obtained by

$$
\Delta R=R_{\mathrm{av}}-\frac{R_{L}}{2} .
$$

Therefore, the range of imaging target in the current HRRP is

$$
R=\Delta R+R_{\text {init }},
$$

where $R_{\text {init }}$ is the initial range of the detected target. The latest first LO delay calculated by the range of imaging target in (4) 


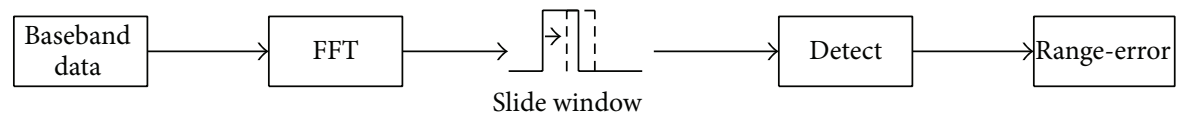

(a)

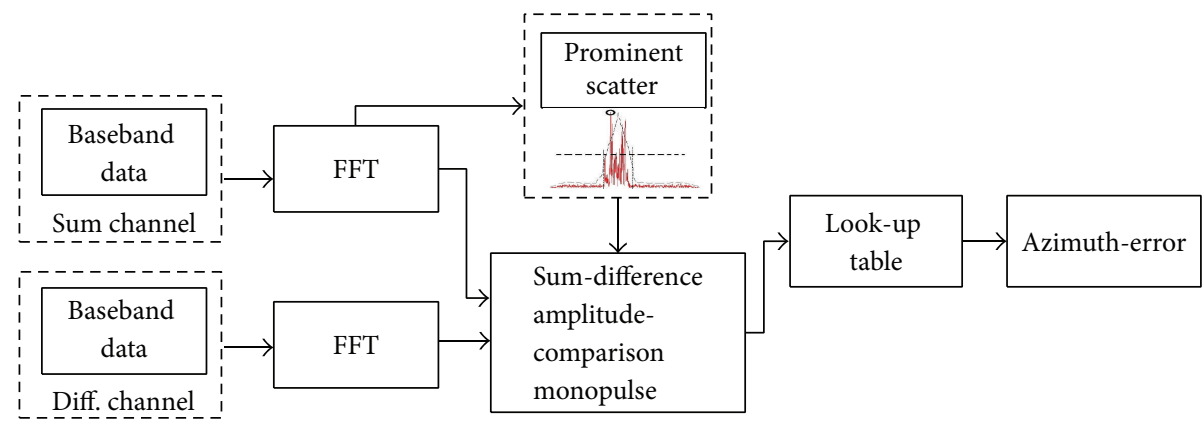

(b)

FIGURE 3: Basic block diagram of range and azimuth tracking. (a) Basic block diagram of range tracking. (b) Basic block diagram of azimuth tracking.

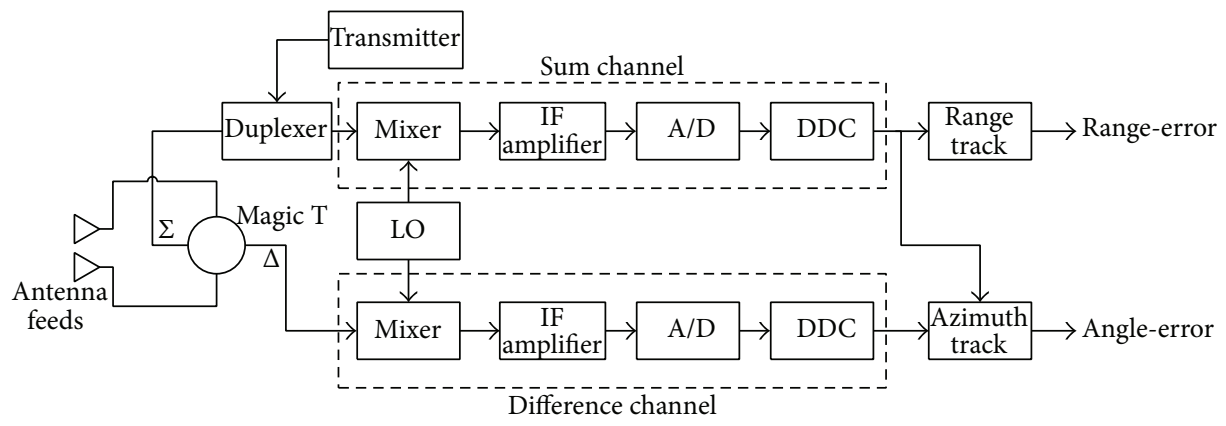

FIGURE 4: Block diagram of the sum-difference amplitude-comparison monopulse in azimuth coordinate.

will be utilized in radar receiver and the sampling gate will be modified adaptively in time series.

2.2.2. Azimuth Tracking. Angle-error is sensed by azimuth tracking for adjusting the LOS of antenna to keep the moving target illuminated permanently. The scatterer with maximum amplitude in the range profile in sum channel is selected for azimuth estimation.

The well-known sum-difference amplitude-comparison monopulse method, as shown in Figure 4, is adopted here to estimate the latest angle-error of the target and adjust the LOS of antenna. Two antenna feeds could be regarded as the simplest phased array system. The sum and difference channels, which are denoted by $\Sigma$ and $\Delta$, respectively, and share a single LO, could be generated by the magic $\mathrm{T}$ module and utilized to sense the angle-error. The first local oscillator time delay, which dictates the selection of the sampling gate, could be calculated from the range-error estimated in sum channel to keep the target located in the center of the imaging range scene. The digital baseband signals of the two channels, which are used in following target tracking and imaging, are obtained from an analogto-digital (A/D) converter and a digital down converter (DDC).
The monopulse ratio, proportional to the angle-error $\Delta \theta$, can be expressed as

$$
\frac{F_{\Delta}(\Delta \theta)}{F_{\Sigma}(\Delta \theta)}=k \cdot \Delta \theta,
$$

where $F_{\Delta}(\Delta \theta)$ and $F_{\Sigma}(\Delta \theta)$ are the difference and the sum patterns, respectively, and $k$ is the monopulse ratio. The estimated azimuth of the target is

$$
\theta=\Delta \theta+\theta_{0}
$$

where $\theta_{0}$ is the current antenna LOS.

In practice, the experimental sum-difference amplitudecomparison monopulse ratios are prestored in the signal processing system. The angle-error will be estimated by searching the look-up table when needed, as shown in Figure 3(b).

\section{Range and Azimuth Tracking Method via Centroid Algorithm}

Owing to the instability of receiver and transient interference, the problem of target echo loss may occur occasionally. In this case, the target may be excluded from the imaging range scene or not be illuminated by antenna due to the erroneous 


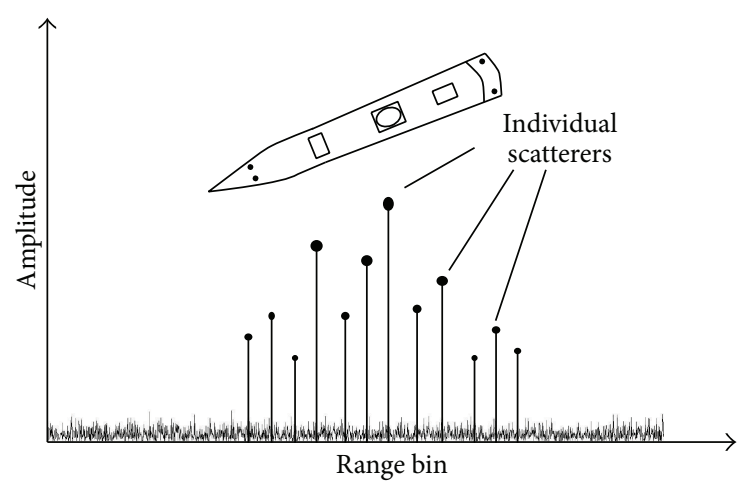

FIGURE 5: Ship and high range resolution profile.

estimations of range and azimuth by RAT method, both of which result in the failure of continuous imaging.

In this section, a modified RATCA method is proposed based on centroid algorithm by using multiple echoes with forgotten factor to improve the performance and robustness of range and azimuth tracking.

3.1. Description of the Centroid Algorithm. The centroid of an object in physics, namely, the center of mass, is the unique point where the weighted relative position of the distributed mass sums to zero. The HRRP of the target can be considered as a single object which consists of several major scatterers with different amplitudes (as shown in Figure 5), which could be utilized in estimating the range and azimuth by amplitude centroiding. Considering that the HRRP of lowvelocity marine target between two successive PRI, which is on the order of hundreds of microseconds, is closely related, the centroid algorithm employed in several successive pulses can be applied to smooth the results and decrease the unexpected interference for the robust tracking performance.

\subsection{Applications of Centroid Algorithm in Range and Azimuth} Tracking. The RAT method generally works well in normal situation. However, some potential risks are involved when sudden jamming and random interference are taken into consideration. The scatterers used for range and azimuth tracking in RAT method are only selected by the corresponding amplitudes, which may be interrupted by sudden outlier. Both range and azimuth tracking will be affected seriously especially for the latter one, which only utilizes one prominent scatterer for angle-error estimation without determining whether the chosen maximum amplitude response is a return from real target or a spurious interference in the same range bin. A modified algorithm named RATCA is proposed here to decrease these impacts of amplitude weighting.

The RATCA method consists of inner-pulse processing and pulse-to-pulse processing. The inner-pulse processing first employs $N$ prominent scatterers for accurate range and azimuth estimations in a single snapshot. Considering that the PRI is hundreds of microseconds, the motion of marine target can be neglected because the range shift is less than the half of range bin length and the change of azimuth is less than the half of antenna beam width during this period. Therefore, the LOS and the first LO time delay are often modified in a fixed tracking interval (typically about 1 second in the practical ISAR) which is much longer than the PRI. The pulse-to-pulse processing can decrease the impact of echo missing by reprocessing with $M$ pulses and is also helpful to decorrelate the sea clutter and interference. $M$ pulses could be chosen in a given interval, which is the inner-pulse interval (IPI) with typical value of 0.1 seconds in the practical ISAR.

3.2.1. Inner-Pulse Processing. Similar to the RAT method, assume that $N$ prominent scatterers in each of the total $M$ target range profiles are selected. The $j$ th HRRP is investigated as a representative, $j=1,2, \ldots, M$. The estimates of range-error and angle-error after inner-pulse processing, $\Delta \widehat{R}_{j}$ and $\Delta \widehat{\theta}_{j}$, can be calculated by centroid algorithm using the data of $N$ selected scatterers:

$$
\left[\begin{array}{c}
\Delta \widehat{R}_{j} \\
\Delta \widehat{\theta}_{j}
\end{array}\right]=\left[\begin{array}{c}
\Delta \mathbf{R}_{j} \\
\Delta \boldsymbol{\theta}_{j}
\end{array}\right] \overline{\mathbf{A}}_{j}^{\mathrm{T}},
$$

where

$$
\begin{aligned}
\Delta \mathbf{R}_{j} & =\left[\begin{array}{llll}
\Delta R_{1 j} & \Delta R_{2 j} & \cdots & \Delta R_{N j}
\end{array}\right], \\
\Delta \boldsymbol{\theta}_{j} & =\left[\begin{array}{llll}
\Delta \theta_{1 j} & \Delta \theta_{2 j} & \cdots & \Delta \theta_{N j}
\end{array}\right], \\
\overline{\mathbf{A}}_{j} & =\left[\begin{array}{llll}
\frac{A_{1 j}}{A_{j \text {-sum }}} & \frac{A_{2 j}}{A_{j \text {-sum }}} & \cdots & \frac{A_{N j}}{A_{j \text {-sum }}}
\end{array}\right], \\
A_{j \text {-sum }} & =\sum_{i=1}^{N} A_{i j} .
\end{aligned}
$$

The range-error, angle-error, and amplitude in sum channel of each selected scatterer are $\Delta R_{i j}, \Delta \theta_{i j}$, and $A_{i j}$, respectively, where $i=1,2, \ldots, N$ represents the order of scatterers used for parameter estimation in each pulse and $j$ denotes the order of pulses. Meanwhile, the estimated amplitude $\widehat{A}_{j}$ is the arithmetic mean amplitude of the $N$ scatterers:

$$
\widehat{A}_{j}=\frac{A_{j \text {-sum }}}{N} .
$$

According to the experimental results, the ratio of the maximum amplitude of the range profile in sum channel with target to that without target is about 30 to $40 \mathrm{~dB}$, as shown in Figure 6.

3.2.2. Pulse-to-Pulse Processing. As mentioned above, the significant advantage of pulse-to-pulse processing is to improve the tracking performance by decreasing the unexpected interference. Because the motion of the marine target is complicated, the significance of historic results should be considered in pulse-to-pulse processing. Therefore, the forgotten factors are used to represent the influence of historic result on the present one, which can be regarded as processing through weighted summation. This principle is illustrated in Figure 7, where the forgotten factors of the jth HRRP are 

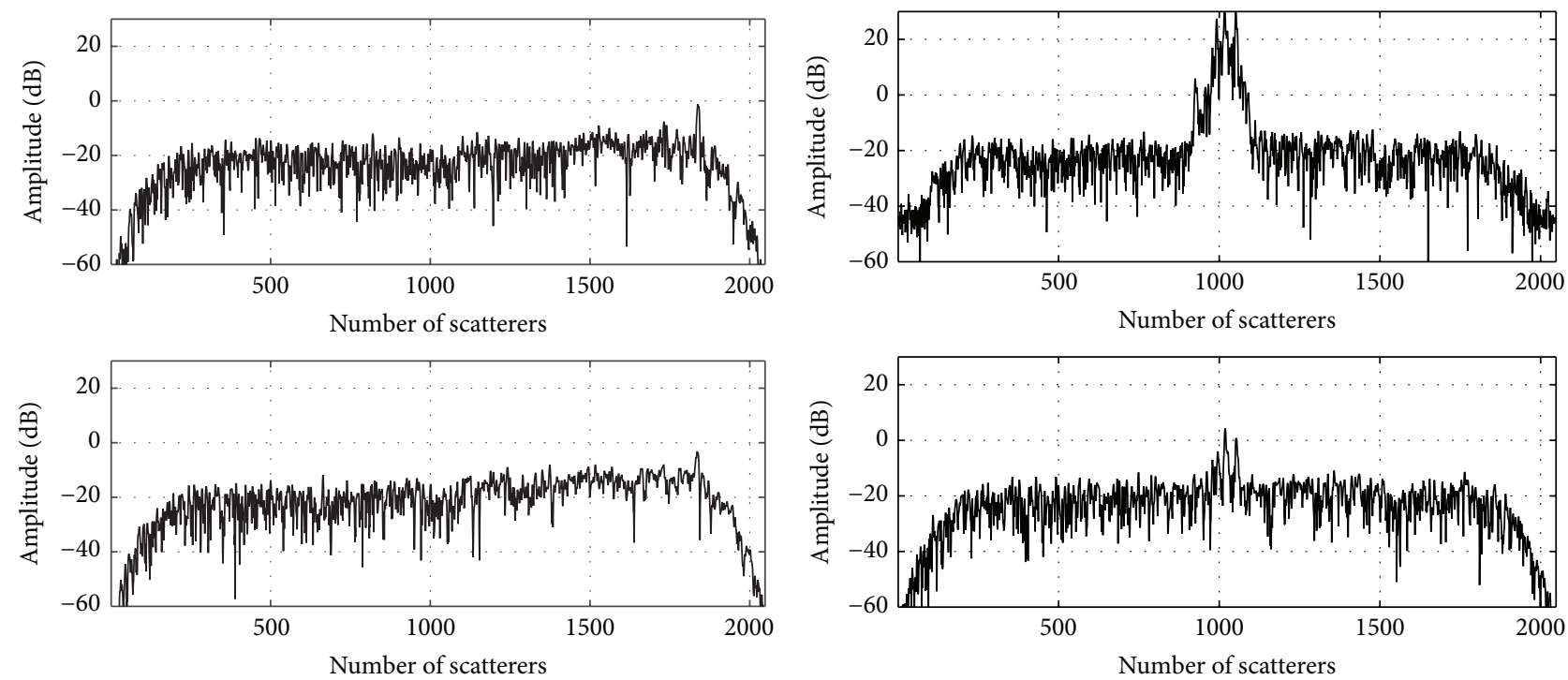

(a)

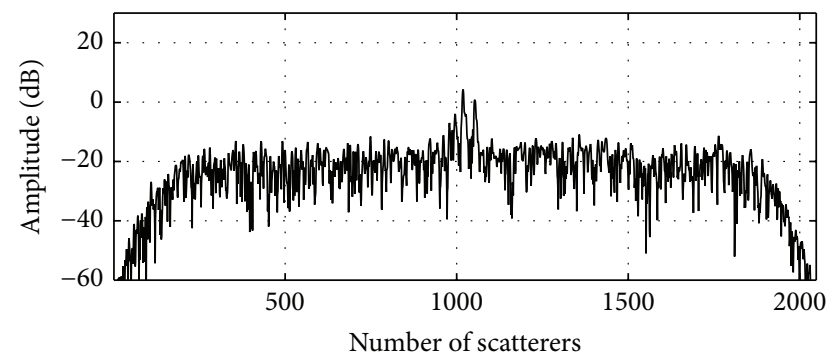

(b)

FIGURE 6: Comparison of range profiles in sum channel (top) and difference channel (bottom) for target missing and existing. (a) Range profiles without targets. (b) Range profiles of the target.

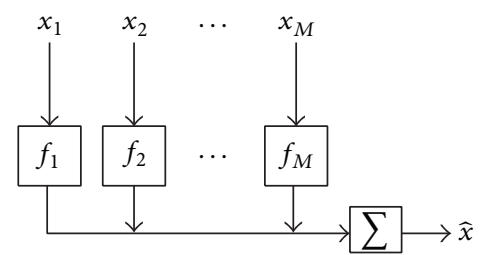

FIGURE 7: Basic principle of the forgotten factor.

represented by the weighted coefficients $f_{i}, i=1 \sim M$, with $\sum_{i=1}^{M} f_{i}=1$. The estimated result with forgotten factor is

$$
\widehat{x}=\mathbf{x} \cdot \mathbf{f}^{\mathrm{T}},
$$

where

$$
\begin{aligned}
& \mathbf{x}=\left[\begin{array}{llll}
x_{1} & x_{2} & \cdots & x_{M}
\end{array}\right], \\
& \mathbf{f}=\left[\begin{array}{llll}
f_{1} & f_{2} & \cdots & f_{M}
\end{array}\right],
\end{aligned}
$$

$x_{i}, i=1,2, \ldots, M$, is the range-error or angle-error estimation after inner-pulse processing.

In the pulse-to-pulse processing with a given length $M$, the latest pulse will be processed by centroid algorithm with the historic $M-1$ pulses with the forgotten factors. Real-time estimations of range-error and angle-error, $\Delta \widehat{R}$ and $\Delta \widehat{\theta}$, are, respectively, given by

$$
\left[\begin{array}{c}
\Delta \widehat{R} \\
\Delta \widehat{\theta}
\end{array}\right]=\frac{1}{\alpha} \cdot\left[\begin{array}{c}
\Delta \widehat{\mathbf{R}} \\
\Delta \widehat{\boldsymbol{\theta}}
\end{array}\right] \cdot \widehat{\mathbf{A}} \cdot \mathbf{f}^{\mathrm{T}},
$$

where

$$
\begin{aligned}
\alpha & =\mathbf{I}_{1 \times M} \cdot \widehat{\mathbf{A}} \cdot \mathbf{f}^{\mathrm{T}}, \\
\Delta \widehat{\mathbf{R}} & =\left[\begin{array}{llll}
\Delta \widehat{R}_{1} & \Delta \widehat{R}_{2} & \cdots & \Delta \widehat{R}_{M}
\end{array}\right], \\
\Delta \widehat{\boldsymbol{\theta}} & =\left[\begin{array}{llll}
\Delta \widehat{\theta}_{1} & \Delta \widehat{\theta}_{2} & \cdots & \Delta \widehat{\theta}_{M}
\end{array}\right], \\
\widehat{\mathbf{A}} & =\operatorname{diag}\left(\widehat{A}_{1}, \widehat{A}_{2}, \ldots, \widehat{A}_{M}\right) .
\end{aligned}
$$

The basic block diagram of RATCA algorithms is shown in Figure 8.

3.3. Types of Forgotten Factors. The weight of historic estimation in each of the target echoes is determined by forgotten factor, which is usually constant, linear, or exponential sequence. Target tracking with RATCA method consists of range tracking and azimuth tracking. For convenience, selection of forgotten factors in range tracking is discussed as a representative. Assume that the radial motion of marine target can be modeled as

$$
R_{i}=R_{\text {init }}+v \cdot i T+\frac{1}{2} a(i T)^{2}
$$

where $i$ is the pulse order in total $M$ IPIs and $i=1,2, \ldots, M$, $R_{\text {init }}$ is the initial radial range to the radar, $v$ and $a$ are target radial velocity and acceleration, respectively, and $T$ is the IPI. For convenience, we assume that

$$
R_{\text {init }}=\frac{R_{L}}{2} .
$$

Thus, the range-error only relates to the target velocity, acceleration, pulse order, and IPI. As mentioned in (12), 


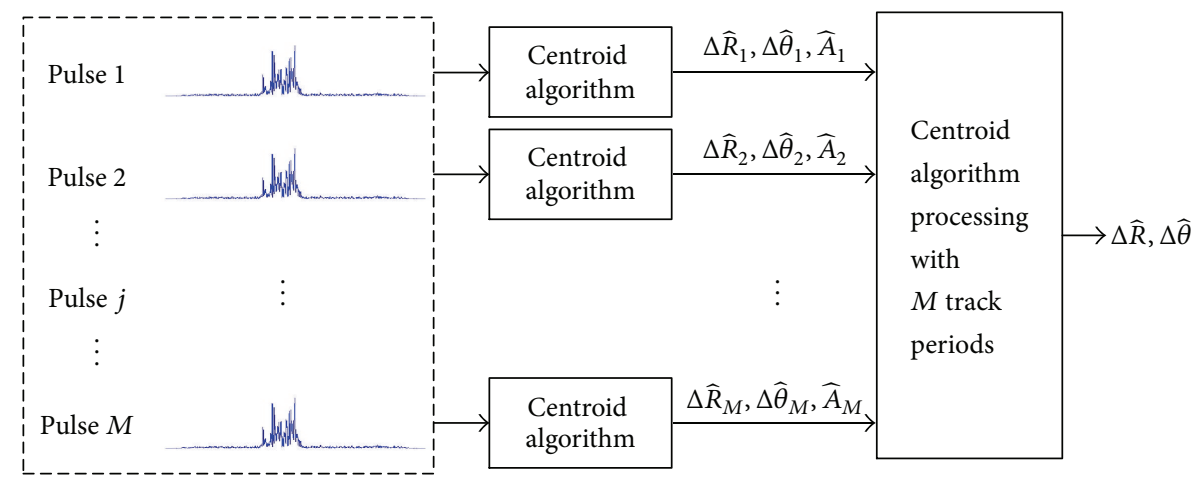

Figure 8: Basic block diagram of RATCA algorithms.

the range-error estimate of the target by performing with $M$ target HRRPs is

$$
\begin{aligned}
\Delta \widehat{R}= & \frac{\sum_{i=1}^{M} \Delta \widehat{R}_{i} A_{i} f_{i}}{\sum_{i=1}^{M} A_{i} f_{i}} \\
= & v T \frac{\sum_{i=1}^{M} i A_{i} f_{i}}{\sum_{i=1}^{M} A_{i} f_{i}}+\frac{1}{2} a T^{2} \frac{\sum_{i=1}^{M} i^{2} A_{i} f_{i}}{\sum_{i=1}^{M} A_{i} f_{i}} \\
& +\frac{\sum_{i=1}^{M} n_{i} A_{i} f_{i}}{\sum_{i=1}^{M} A_{i} f_{i}}
\end{aligned}
$$

where $\Delta \widehat{R}_{i}$ and $n_{i}$ are the range-error and noise in each inner-pulse processing, respectively. Assuming that the target amplitude keeps invariant during the processing, the above can be simplified to

$$
\Delta \widehat{R}=v T \frac{\sum_{i=1}^{M} i f_{i}}{\sum_{i=1}^{M} f_{i}}+\frac{1}{2} a T^{2} \frac{\sum_{i=1}^{M} i^{2} f_{i}}{\sum_{i=1}^{M} f_{i}}+\frac{\sum_{i=1}^{M} n_{i} f_{i}}{\sum_{i=1}^{M} f_{i}} .
$$

Note that the true value of the range-error in the $M$ th IPI without pulse-to-pulse processing is

$$
\Delta R_{M}=M v T+\frac{1}{2} a M^{2} T^{2}
$$

The true range-error in the above is utilized as the reference value for the following analysis with different types of forgotten factors.

3.3.1. "Pulse" Type. Under this condition, the forgotten factors can be expressed as

$$
f_{i}= \begin{cases}0 & i=1, \ldots, M-1 \\ 1 & i=M\end{cases}
$$

This is the simplest type and the estimate after pulseto-pulse processing is simplified to the form in (18). The estimation of range-error could be expressed as

$$
\Delta \widehat{R}_{P}=M v T+\frac{1}{2} a M^{2} T^{2}+n_{M} .
$$

The above satisfies the real position ideally while suffering the same level of noise background. The estimation error is given as

$$
\operatorname{err}_{P}(M, v, a, T)=\Delta \widehat{R}_{P}-\Delta R_{M}=n_{M}
$$

3.3.2. "Constant" Type. In this type, the forgotten factors turn to be

$$
f_{i}=\frac{1}{M}, \quad i=1,2, \ldots, M
$$

and the output estimate is

$$
\begin{aligned}
\Delta \widehat{R}_{C}= & \frac{M+1}{2} v T+\frac{1}{2} a T^{2} \frac{(M+1)(2 M+1)}{6} \\
& +\frac{\sum_{i=1}^{M} n_{i}}{M} .
\end{aligned}
$$

The estimation error is

$$
\begin{aligned}
\operatorname{err}_{C}(M, v, a, T)= & \Delta \widehat{R}_{C}-\Delta R_{M} \\
= & \frac{1-M}{2} v T \\
& +\frac{1}{2} a T^{2} \frac{(4 M+1)(1-M)}{6} \\
& +\frac{\sum_{i=1}^{M} n_{i}}{M} .
\end{aligned}
$$

3.3.3. "Linear" Type. Under this condition, the style of forgotten factors is a linear function with the form

$$
f_{i}=k_{s} i, \quad i=1,2, \ldots, M .
$$

The value of the slope $k_{s}$ is $2 / M(M+1)$, subject to $\sum_{i=1}^{M} f_{i}=1$. The estimate can be calculated as

$$
\begin{aligned}
\Delta \widehat{R}_{L}= & \frac{2 M+1}{3} v T+\frac{1}{2} a T^{2} \frac{M(M+1)}{2} \\
& +\frac{2}{M(M+1)} \sum_{i=1}^{M} i \cdot n_{i} .
\end{aligned}
$$


Similarly, the estimation error is

$$
\begin{aligned}
\operatorname{err}_{L}(M, v, a, T)= & \Delta \widehat{R}_{L}-\Delta R_{M} \\
= & \frac{1-M}{3} v T+\frac{1}{2} a T^{2} \frac{M(1-M)}{2} \\
& +\frac{2}{M(1+M)} \sum_{i=1}^{M} i \cdot n_{i} .
\end{aligned}
$$

3.3.4. "General" Type. "General" means that the selection of forgotten factors is undetermined. The estimate of rangeerror, $\Delta \widehat{R}_{G}$, is given in (17) and the estimation error is

$$
\begin{aligned}
\operatorname{err}_{G}(M, v, a, T)= & \Delta \widehat{R}_{G}-\Delta R_{M} \\
= & M v T\left(f_{M}-1\right)+v T \sum_{i=1}^{M-1} i \cdot f_{i} \\
& +\frac{1}{2} a M^{2} T^{2}\left(f_{M}-1\right) \\
& +\frac{1}{2} a T^{2} \sum_{i=1}^{M-1} i^{2} \cdot f_{i}+\sum_{i=1}^{M} n_{i} f_{i} .
\end{aligned}
$$

In fact, the three types of forgotten factors are special cases of "General" type. The IPI $T$ and length of pulse-topulse processing $M$ are often given as a priori knowledge in practical ISAR. Therefore, to minimize the estimation error, the forgotten factors close to the latest RAT results should be dominated with large values of velocity and acceleration. From (28), we can see that the ideal choice is to set $f_{M}=$ 1 and the others to zero. However, this will lead to poor robustness as will be investigated later. An exponential model of forgotten factor for relative high speed and acceleration target is introduced as

$$
f_{i}=\frac{M}{\beta} \cdot e^{i-M}, \quad i=1,2, \ldots, M,
$$

where $\beta=\sum_{i=1}^{M} M e^{i-M}=M\left(e^{1-M}-e\right) /(1-e)$ is the normalized factor.

The range tracking RMSEs with different forgotten factors versus velocity in various SNRs with $a=0 \mathrm{~m} / \mathrm{s}^{2}$ are shown in Figure 9. The velocity of the simulated target is distributed among [ $\left.\begin{array}{ll}1 & 15\end{array}\right] \mathrm{m} / \mathrm{s}$. The SNR of the simulated target varies from 5 to 25 in $\mathrm{dB}$ with step of $10 \mathrm{~dB}$.

When the target velocity is approximately smaller than $5 \mathrm{~m} / \mathrm{s}$, the "Constant" and "Linear" types outperform the other two kinds of forgotten factors. When target velocity increases, the RMSEs of "Constant" and "Linear" types go up rapidly while the "Exponential" one is still stable. Although the radar line of sight and the first LO time delay are modified in a fixed interval longer than the PRI in practice, the radial and rotational motion can be ignored for low-speed marine target. The noise could be averaged via constant- or linear-weighting while the high range resolution profile of the target keeps steady. However, motion of the target at high speed cannot be neglected because the movement may spread through adjacent range cells or azimuth cells. The latest range-error and angle-error may be seriously affected by the historical estimations, which can be relieved properly by exponential weighting. As shown in Figure 9, it is obvious that the "Exponential" type is preferred in tracking highspeed marine target while the "Constant" and "Linear" types show advantages in low-speed target tracking.

The range tracking RMSEs with different accelerations with velocity fixed at $v=1 \mathrm{~m} / \mathrm{s}$ are illustrated in Figure 10, from which a situation similar to that in Figure 9 can be seen. When the acceleration of the target is small, the RMSEs of "Constant" and "Linear" types are smaller than those of the others. With the increasing of target acceleration, the range tracking RMSE of "Exponential" type keeps stable and shows a superior performance to the other three types.

Since azimuth tracking uses the same processing method as used in range tracking, similar performances could be predictable in the azimuth tracking and thus are omitted here to save space.

To investigate the range-error RMSEs estimated of the aforementioned forgotten factors with different SNR, the tracking RMSEs of a typical marine target, which is similar to the cooperative vessel in the experiment referred to in this paper, are shown in Figure 11. It is obvious that the range tracking RMSEs with different forgotten factors decrease monotonically with the increasing of target SNR, with a given velocity or acceleration. To be precise, the "Constant" and "Linear" types of forgotten factors show the better performances among all the four types due to the low speed and acceleration of the target. Thus, the "Linear" type of forgotten factors is selected in the marine experiment.

\section{Simulation and Experimental Results}

In this section, tracking error analysis and simulation results with different kinds of forgotten factors are given versus different target velocities, accelerations, and SNRs. Experimental results from an ISAR marine imaging experiment are provided to verify the proposed method. The system parameters are as follows: radar carrier frequency $f_{0}=$ 11.4 GHz, imaging bandwidth $B_{W}=400 \mathrm{MHz}$, DDC sample rate $f_{s}=28 \mathrm{MHz}$, pulse width $\tau=30 \mu \mathrm{s}$, and PRI $T_{r}=$ $400 \mu$ s. Two experiments are conducted:

(1) Successive radar echoes of a cooperative moving ship were recorded without range or azimuth tracking by an onshore radar to verify the performances of RAT and RATCA methods. The results are presented in Sections 4.1, 4.2, and 4.3.

(2) Locate the radar on a moving vessel platform. The same cooperative moving target was imaged continuously with simultaneous range and azimuth tracking. The ISAR images are given in Section 4.4.

4.1. RATCA and RAT Algorithms in the Normal Situation. If the radar works normally, the wideband echoes of the target could be received correctly. Under this condition, the performance of tracking is mainly related to the interior noise. The estimations of range and azimuth with a unique 


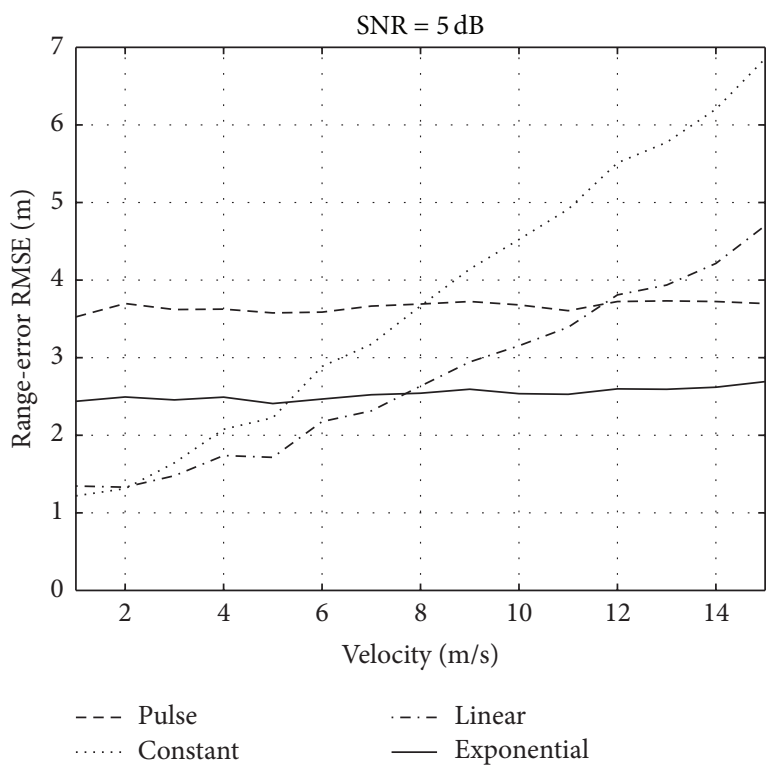

(a)

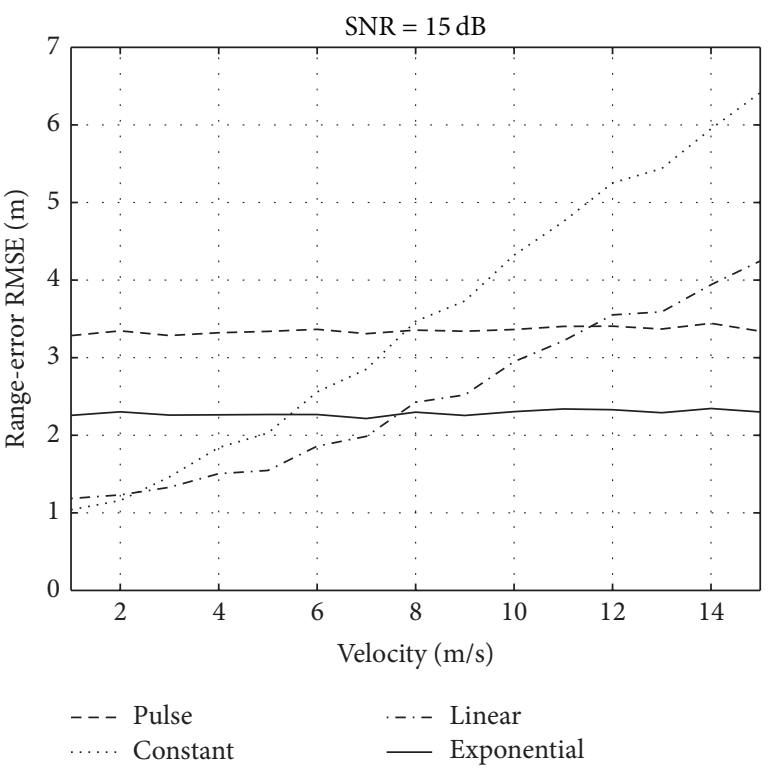

(b)

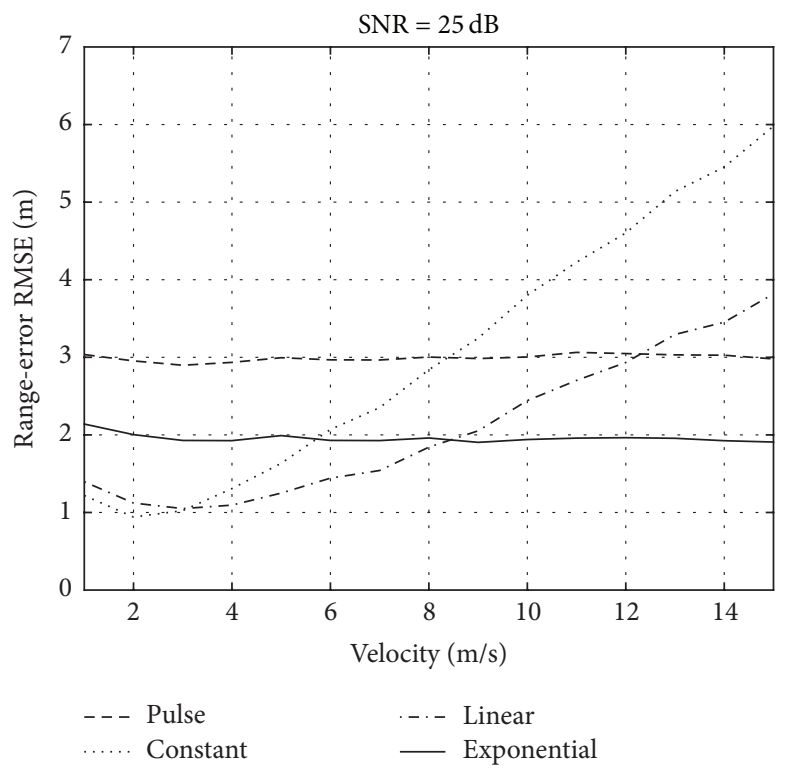

(c)

FIGURE 9: Range-error root-mean-square errors (RMSEs) of different forgotten factors versus velocity with $a=0 \mathrm{~m} / \mathrm{s}^{2}$. (a) SNR = 5dB, (b) $\mathrm{SNR}=15 \mathrm{~dB}$, and $(\mathrm{c}) \mathrm{SNR}=25 \mathrm{~dB}$.

echo fluctuate slightly, assuming that the SNR of the target in sum pattern is coequal to the null depth in difference pattern, though the target and seawater keep a standstill [18]. In actual application, these fluctuations are desired to be minimized to decrease the error caused by mechanical rotation. The experimental results in Figure 12 present the comparison between tracking performances of RAT and RATCA methods.

The length of the cooperative ship is 30 meters and the antenna beam width is 3.3 degrees. As shown in Figure 12, both of the RAT and RATCA methods could work well in the normal situation. It appears that the estimations of rangeerror with RATCA method are smoother than that based on
RAT algorithm, as displayed by the thick line in Figure 12(a). For comparison purpose, a series of range-error estimations without using the modified method are presented by the thin lines. As illustrated in Figure 12(b), the azimuth tracking results are smoother than that without being processed. The impacts introduced by fluctuations of targets and internal noise in radar are decreased; thus, RATCA algorithm shows a better tracking performance than RAT method.

4.2. RATCA and RAT Algorithms in Extreme Situations. In this subsection, the performances of RAT and RATCA in the extreme situation are studied. Two conditions are taken into 


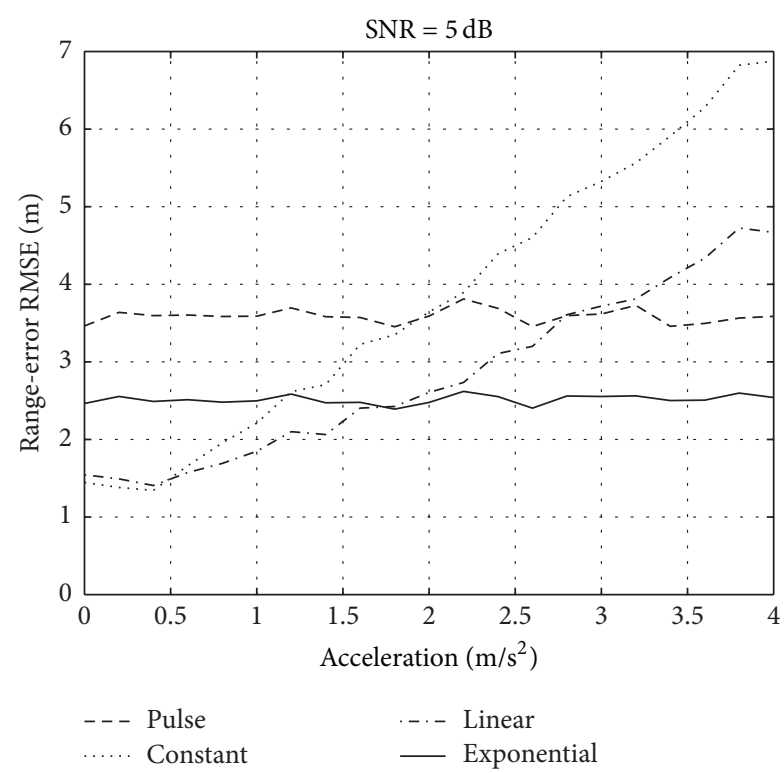

(a)

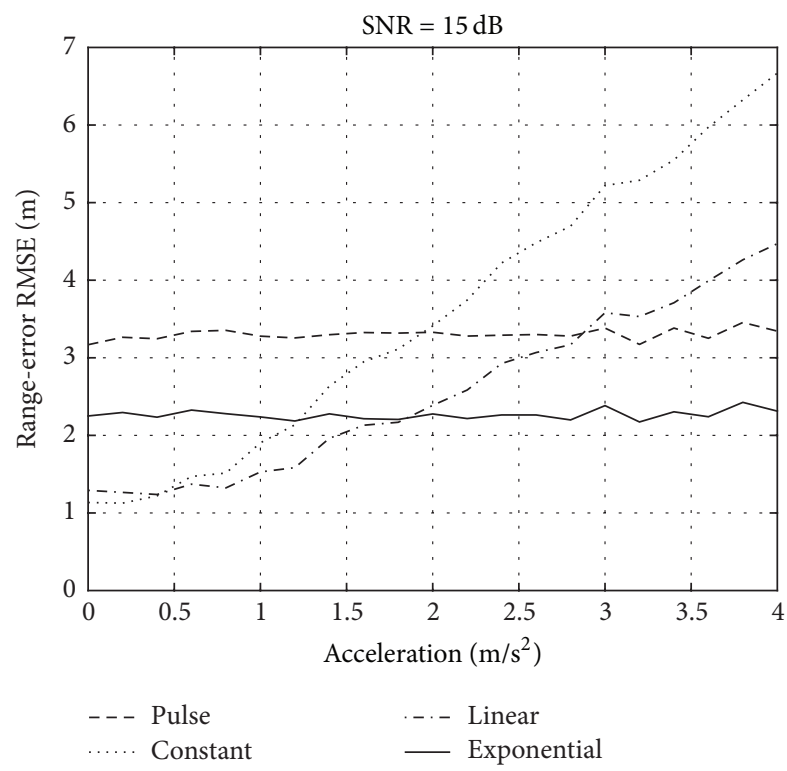

(b)

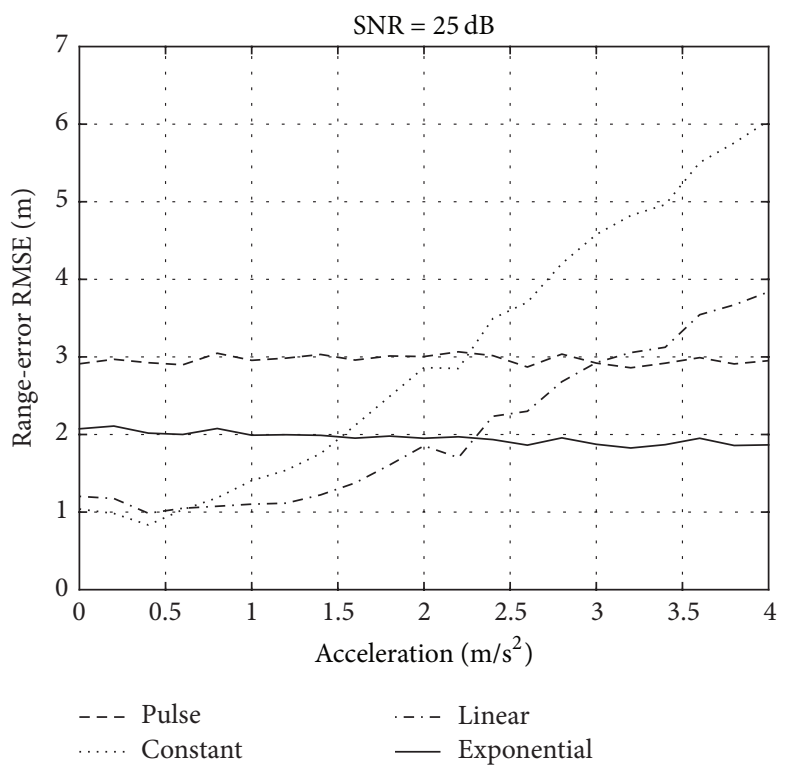

(c)

FIGURE 10: Range-error RMSEs of different forgotten factors versus acceleration with $v=1 \mathrm{~m} / \mathrm{s}$. (a) SNR $=5 \mathrm{~dB}$, (b) $\mathrm{SNR}=15 \mathrm{~dB}$, and (c) $\mathrm{SNR}=25 \mathrm{~dB}$.

consideration: echo loss and jamming existence. The former one is analyzed with experimental data and the latter one is investigated with computer simulations.

The target is generally located in the middle of imaging scene and the center of the antenna beam in normal situation. Once the echo is missing, the maximum erroneous rangeerror may deviate from the real one for about $105 \mathrm{~m}$. The value of the deviation depends on the practical radar parameters and affects the first LO time delay. Once the range-error estimation exceeds the upper or nether threshold, the range tracking will fail. As shown in Figure 13(a), many erroneous range-error estimates, which exceed the threshold obviously, are obtained by RAT method; based on the RATCA method, the range-error estimation is revealed to be precise and stable.

In azimuth tracking with the RAT method, if only the estimated angle-error exceeds 1.65 degrees, which is a half of the practical antenna $3 \mathrm{~dB}$ beam width (only 3.3 degrees in the practical ISAR) and is presented as upper and nether thresholds in Figure 13(b), the target will not be illuminated by antenna main beam immediately. Therefore, the ISAR imaging will fail. On the contrary, when the proposed RATCA method is used, the impact caused by echo missing is significantly relieved, as shown in Figure 13(b). 


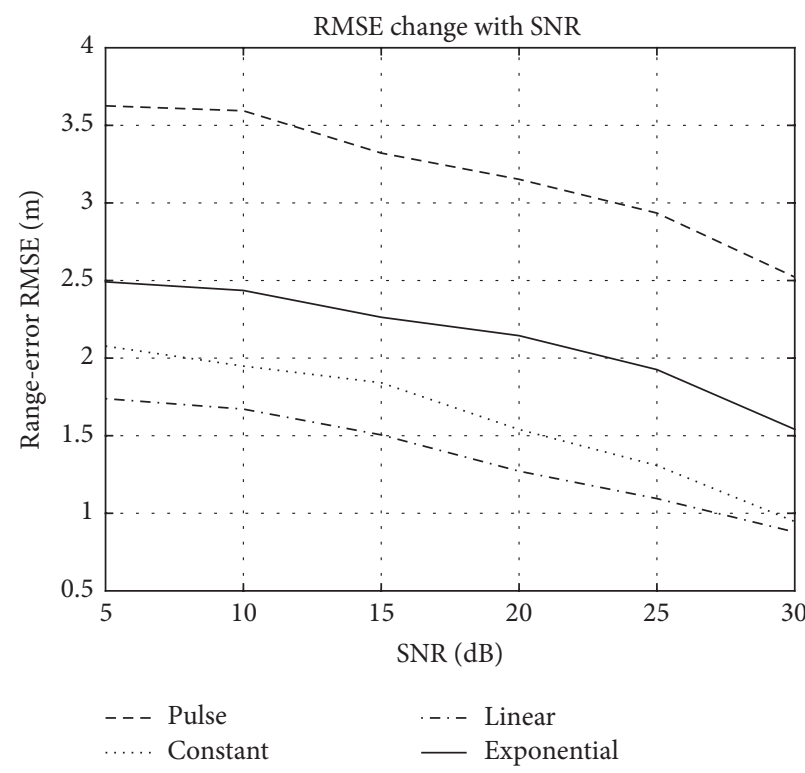

(a)

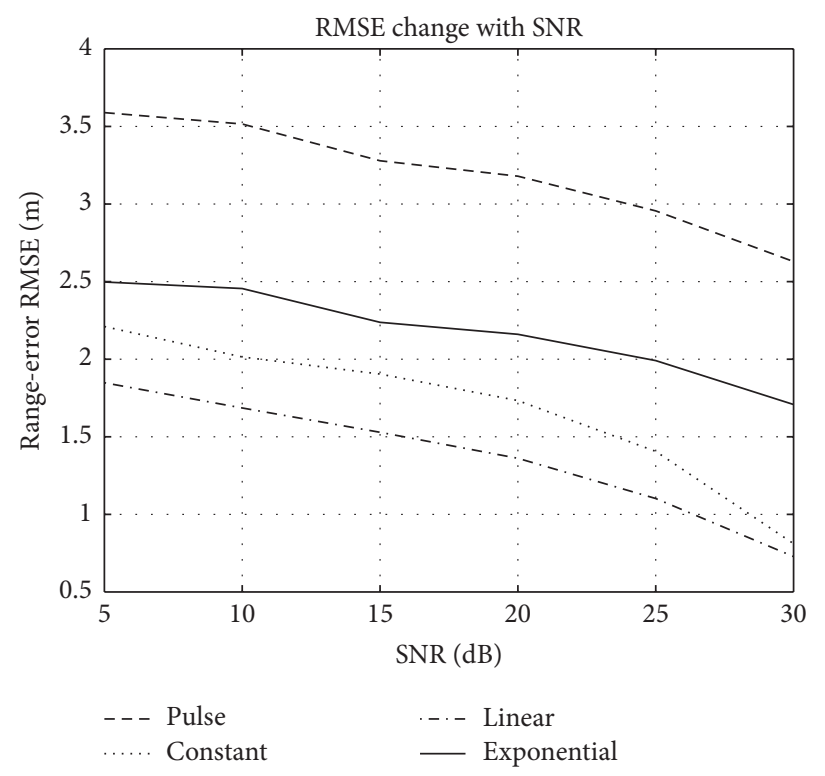

(b)

FIGURE 11: Range-error RMSEs in typical marine target parameters. (a) RMSE versus SNR with $v=4 \mathrm{~m} / \mathrm{s}$ and $a=0 \mathrm{~m} / \mathrm{s}^{2}$. (b) RMSE versus SNR with $a=1 \mathrm{~m}^{2} / \mathrm{s}$ and $v=1 \mathrm{~m} / \mathrm{s}$.

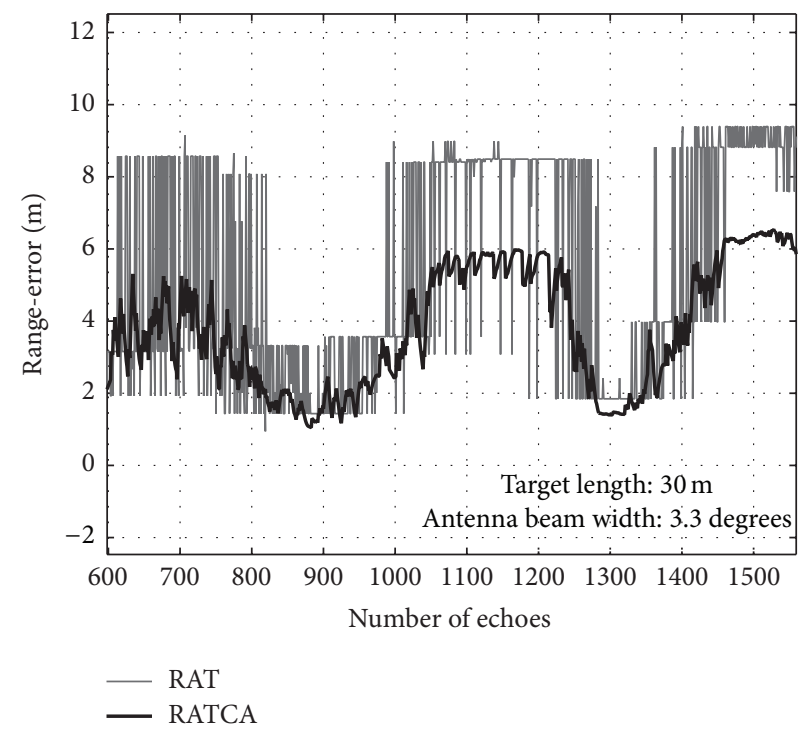

(a)

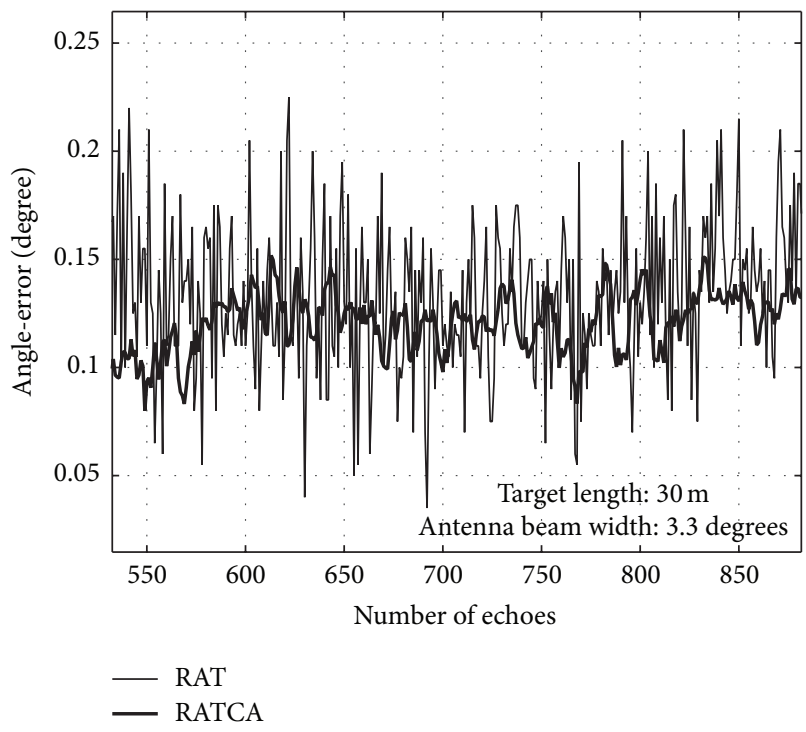

(b)

FiguRE 12: Comparisons of tracking performances between RAT and RATCA when radar is performing normally. (a) Range-error. (b) Azimuth-error.

Figures 13(c) and 13(d) illustrate the tracking results of RAT and RATCA methods with the existence of jamming at one edge of imaging scene, which means that the erroneous estimation of range-error will be much larger than the set value. The predetermined range-error and angle-error of simulated target are $0 \mathrm{~m}$ and -0.26 degrees, respectively. The jamming has the same SNR with the simulated target and appears once per 64 pulses.

The range and azimuth tracking results show that both of the range-error and angle-error exceed the threshold obviously with RAT method while RATCA method still works robustly.

4.3. Robustness and Bias of RATCA Algorithm. According to the experimental results above, it is obvious that if echoes from imaging target are correctly received, the target will be tracked accurately and located in the middle of the imaging scene and antenna beam. The RATCA algorithm still works well though the random noise is dominated when the target echo is missing and the RAT algorithm fails. In this 


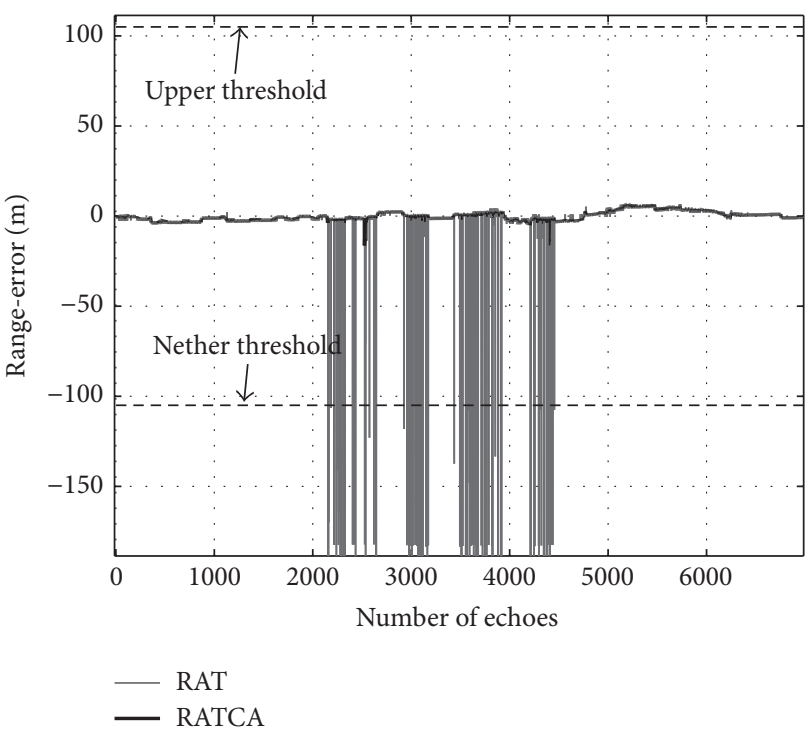

(a)

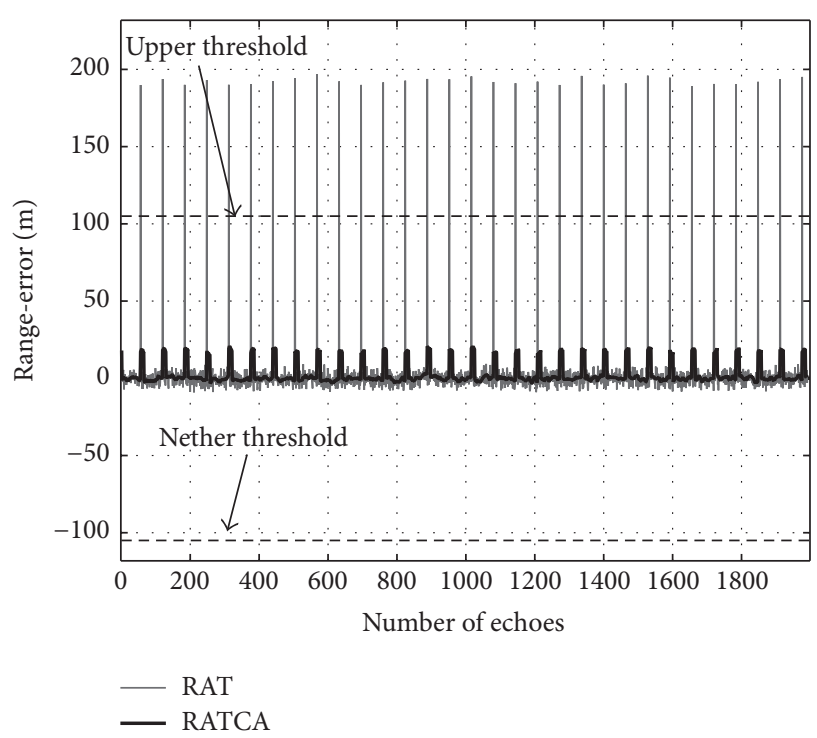

(c)

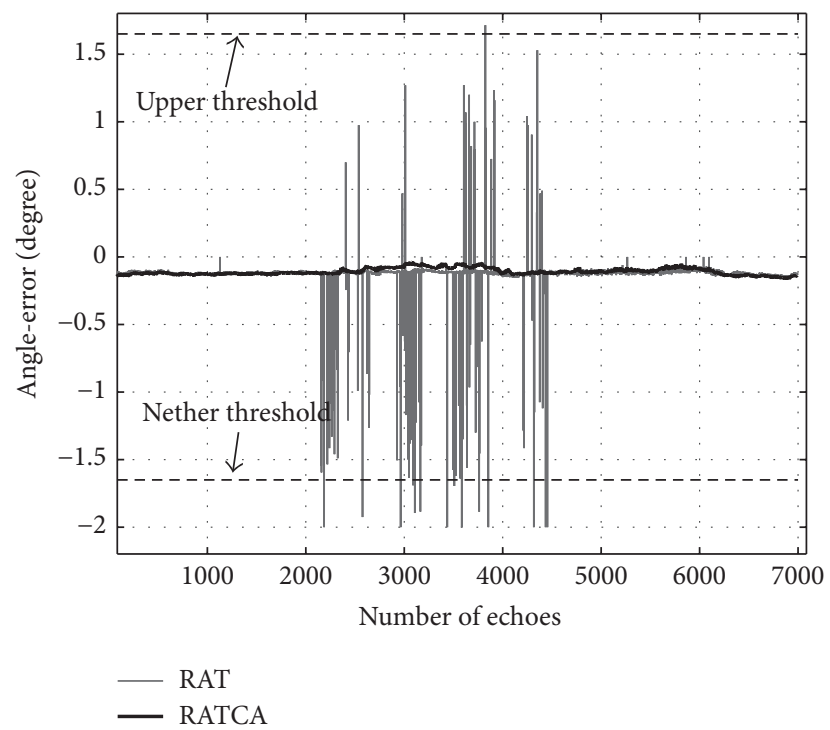

(b)

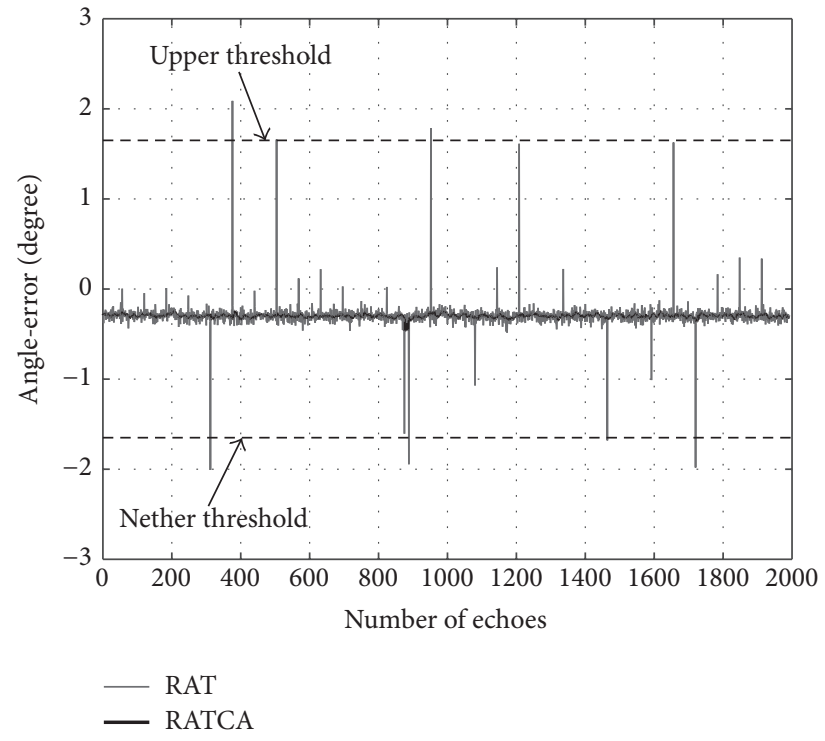

(d)

FIGURE 13: Tracking performances of the RAT and RATCA method in extreme condition. (a) Range estimations in RAT and RATCA methods with echo loss. (b) Azimuth estimations in RAT and RATCA methods with echo loss. (c) Range estimations in RAT and RATCA methods with jamming. (d) Azimuth estimations in RAT and RATCA methods with jamming.

part, the robustness of RATCA algorithm is theoretically analyzed. Considering the influence induced by the weighted historical estimations with different forgotten factors, the bias of RATCA estimator is also investigated.

4.3.1. Robustness. The adjustment of the first LO time delay and rotation of antenna will be slight when echoes are received correctly because the real values of range-error $\Delta R_{\text {true }}$ and angle-error $\Delta \theta_{\text {true }}$ are very small [18]. The target will be tracked continuously if the values of range-error and angle-error are smaller than the tracking thresholds of range and azimuth, $R_{T}$ and $\theta_{T}$, respectively. In general, $R_{T}=R_{L} / 3$ and $\theta_{T}=\theta_{3 \mathrm{~dB}} / 2$, where $\theta_{3 \mathrm{~dB}}$ is antenna beam width and $R_{L}$ is the width of the imaging scene, which is a function of the DDC sample rate $f_{s}$, transmitting bandwidth $B_{W}$, pulse width $\tau$, and the speed of light $c$ :

$$
R_{L}=\frac{c \times \tau \times f_{s}}{2 B_{W}} .
$$

Due to the erroneous estimations of range and azimuth induced by echo missing, the first LO time delay and LOS of antenna will be modified by mistake. Assume that $\Delta R_{\text {false }}$ is the maximum estimated range-error induced by echo missing, which is half of the imaging scene width. Denote by $\Delta \theta_{\text {false }}$ the maximum estimated angle-error when echo is missing, which is usually half of the antenna beam width. 
The performances of range-error and angle-error estimations with "Constant" and "Linear" forgotten factors are similar when target SNR is higher than $30 \mathrm{~dB}$ as shown in Figure 11. Thus, the robustness of RATCA method with "Constant" forgotten factors is analyzed for convenience. Suppose that $k$ pulses are missing during the tracking interval consisting of total $M$ pulses and the target is aligned with the antenna. Under the worst condition, to obtain an acceptable estimation of range by using RATCA algorithm, it is required that

$$
\frac{\Delta R_{\text {false }} \times k \times A_{\text {false }}+\Delta R_{\text {true }} \times(M-k) \times A_{\text {true }}}{k \times A_{\text {false }}+(M-k) \times A_{\text {false }}}<R_{T},
$$

where $A_{\text {false }}$ and $A_{\text {true }}$ are the maximum amplitudes in range profile with or without target, respectively, $R_{T}=$ $R_{L} / 3, \Delta R_{\text {false }}=R_{L} / 2$, and $\Delta R_{\text {true }}$ is very small. The ratio of the maximum amplitude in range profile of target to that when the target echo is missing will be 30 to $40 \mathrm{~dB}$ (denoted by $G$ in $\mathrm{dB}$ ), as shown in Figure 6. Therefore, the maximum amplitude of range profile $A_{\text {true }}$ when radar operates normally can be given by

$$
A_{\text {true }}=A_{\text {false }} \times 10^{G / 20} \text {. }
$$

Substituting (32) into (31), the inequality can be equivalently expressed as

$$
k<\frac{10^{G / 20}}{10^{G / 20}+0.5} \times M
$$

Recalling that the value of $G$ is large enough, (33) can be simplified as

$$
k<M \text {. }
$$

Similar to (31), the requirement to obtain an acceptable azimuth estimation is given by

$$
\frac{\Delta \theta_{\text {false }} \times k \times A_{\text {false }}+\Delta \theta_{\text {true }} \times(M-k) \times A_{\text {true }}}{k \times A_{\text {false }}+(M-k) \times A_{\text {true }}}<\theta_{T},
$$

where $\Delta \theta_{\text {false }}=\theta_{3 \mathrm{~dB}} / 2, \theta_{T}=\theta_{3 \mathrm{~dB}} / 2$, and $\Delta \theta_{\text {true }}$ is very small; (35) can be simplified to

$$
k<M \text {. }
$$

From (34) and (36), it can be inferred that the range and azimuth tracking can be accomplished with at least one pulse received correctly during tracking interval with $M$ pulses.

4.3.2. Bias. In this subsection, the biases of estimations with four types of forgotten factors are investigated with typical parameters of a marine target. Assume that the estimator operates in Gaussian white noise with zero mean.

(i) "Pulse" Type. As mentioned before, the forgotten factors can be expressed in (19). Under this condition, the estimations of range-error by RATCA are simplified to the form in (18), which satisfies the real position ideally while suffering the same level of noise error. The pulse-to-pulse processing is degenerated into a point estimate method. The bias of RATCA estimator with forgotten factor of "Pulse" type is

$$
b_{P}=E\left[\Delta \widehat{R}_{P}-\Delta R_{M}\right]=E\left[n_{M}\right]=0,
$$

where $\Delta \widehat{R}_{P}$ and $\Delta R_{M}$ are the estimated range-error and true value of range-error, respectively, and $n_{M}$ is Gaussian white noise in the $M$ th pulse. Under this condition, RATCA estimator has the simplest form and is unbiased.

(ii) "Constant" Type. The form of forgotten factor in "Constant" type is shown in (22). Similarly, we obtain the bias of RATCA estimator under this condition as

$$
\begin{aligned}
& b_{C}(M, v, a, T)=E\left[\Delta \widehat{R}_{C}-\Delta R_{M}\right]=E\left[\frac{1-M}{2} v T\right. \\
& \left.+\frac{1}{2} a T^{2} \frac{(4 M+1)(1-M)}{6}+\frac{\sum_{i=1}^{M} n_{i}}{M}\right]=\frac{1-M}{2} \\
& \cdot v T+\frac{1}{2} a T^{2} \frac{(4 M+1)(1-M)}{6} .
\end{aligned}
$$

The value of (38) is nonzero and connected with $M$, velocity and acceleration of target, and IPI. With a given list of parameters, the RATCA estimator has a known bias. However, the information of target may vary. To analyze the availability of RATCA estimator, the bias in (38) is calculated with typical parameters of marine target.

Considering the fact that IPI is fixed and typically about 0.1 seconds in the practical ISAR, we are interested in the maximum number of $M$ in tolerable bias. As mentioned before, the range tracking threshold $R_{T}$ is about $105 \mathrm{~m}$ in the experimental ISAR; thus, the range tracking is available if the tolerable bias satisfies

$$
\left|b_{C}(M, v, a, T)\right|_{T=0.1}<105 \text {. }
$$

Assuming that the parameters of the targets are identical to those of Section $3.3\left(v_{\max }=15 \mathrm{~m} / \mathrm{s}, a_{\max }=4 \mathrm{~m} / \mathrm{s}^{2}\right)$, we have

$$
M<73 .
$$

(iii) "Linear" Type. The form of forgotten factor in "Linear" type is shown in (25). The bias of RATCA estimator under this condition is

$$
\begin{aligned}
& b_{L}(M, v, a, T)=E\left[\Delta \widehat{R}_{L}-\Delta R_{M}\right]=E\left[\frac{1-M}{3} v T\right. \\
& \left.+\frac{1}{2} a T^{2} \frac{M(1-M)}{2}+\frac{2}{M(1+M)} \sum_{i=1}^{M} i \cdot n_{i}\right] \\
& =\frac{1-M}{3} v T+\frac{1}{2} a T^{2} \frac{M(1-M)}{2} .
\end{aligned}
$$


Similarly, the bias is tolerable if

$$
M<82
$$

which is also accepted in practical ISAR.

(iv) "Exponential" Type. The form of forgotten factor in "Exponential" type is shown in (29). The bias of RATCA estimator under this condition can be expressed as

$$
\begin{aligned}
& b_{E}(M, v, a, T)=E\left[\Delta \widehat{R}_{E}-\Delta R_{M}\right] \\
& =E\left[M v T\left(f_{M}-1\right)+v T \sum_{i=1}^{M-1} i \cdot f_{i}\right. \\
& \left.\quad+\frac{1}{2} a M^{2} T^{2}\left(f_{M}-1\right)+\frac{1}{2} a T^{2} \sum_{i=1}^{M-1} i^{2} \cdot f_{i}+\sum_{i=1}^{M} n_{i} f_{i}\right] \\
& =M v T\left(\frac{1-e}{e^{1-M}}-1\right)+v T \cdot L_{1}+\frac{1}{2} a T^{2} M^{2}\left(\frac{1-e}{e^{1-M}}\right. \\
& \quad-1)+\frac{1}{2} a T^{2} \cdot L_{2},
\end{aligned}
$$

where $L_{1}=\sum_{i=1}^{M-1} i \cdot f_{i}=\left((1-e) /\left(e^{1-M}-e\right)\right) \sum_{i=1}^{M-1} i \cdot e^{i-M}$ and $L_{2}=\sum_{i=1}^{M-1} i^{2} \cdot f_{i}=\left((1-e) /\left(e^{1-M}-e\right)\right) \sum_{i=1}^{M-1} i^{2} \cdot e^{i-M} \cdot \Delta \widehat{R}_{E}$ could be estimated with the exponential forgotten factors expressed in (29).

The accepted range of $M$ could be calculated via computer simulation due to the complex form of (43) as

$$
M<4475 \text {. }
$$

Though the maximum value of $M$ seems to be quite different from those in (40) and (42), it is reasonable because the weighting of the latest estimation in total $M$ IPIs, $f_{M}$, is significantly larger than the historical $M-1$ ones in the forgotten factor of "Exponential" type.

When a target moves along the tangential direction of radar antenna LOS, its azimuth changes most rapidly, which means that azimuth tracking is more difficult. If (14) is utilized for tangential motion, similar results for azimutherror and its bias are anticipated because a small arc length can be approximated by a straight line. Since the length of arc with arc-angle 1.65 degrees and radius $3.65 \mathrm{~km}$ is 105 meters, the restriction on $M$ resulting from azimuth-error and azimuth tracking threshold would be looser than that of range dimension only if radial range of the target from radar is more than $3.65 \mathrm{~km}$. Therefore, it is reasonable to predict that the results of azimuth tracking, which have been omitted here, are similar to those of the range tracking in a list of predetermined parameters because the same processing is utilized. Considering the number of $M$ is usually 10, though these analyses indicate that the selection of forgotten factor influences the estimation bias of RATCA method, the RATCA estimator works effectively in most radar parameters.

4.4. Experimental Results of Continuous Imaging. The marine experiment was conducted in January 2014 at the Yellow Sea, China. As shown in Figure 14, the route of the vessel

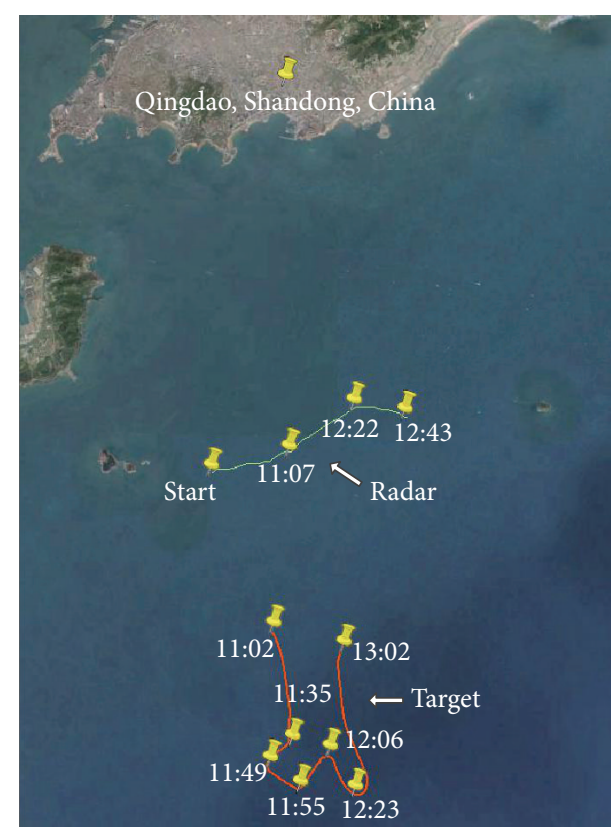

FIGURE 14: Routes of radar platform and cooperative target.

where the radar locates is green-colored. The course of the corresponding cooperative ship target is plotted in red, which has complex maneuverability during the experiment. Time stamps are available and helpful to make a comparison between ISAR images and the real attitude of the target.

During the experiment, a priori knowledge of cooperative target, such as range and azimuth relative to the antenna line of sight, is estimated first in detecting mode. Then, the imaging mode turns to work and the ISAR images of the target could be obtained continuously. There is no requirement of any redetection because the latest target range and azimuth, which is curial to data record and target capture, are obtained successively and simultaneously by range and azimuth tracking.

As investigated in Section 4.2, under some extreme condition, the maximum erroneous range-error and angleerror may exceed the acceptable limits with RAT method; thus, the antenna LOS and sampling gate will be adjusted erroneously. The imaging target will be lost and the following ISAR imaging processing fails. Therefore, the redetection is forced to be carried out, which would be a waste of time, and the ability to monitor some threatening target is limited. Considering some potential risks such as sudden jamming and random interference, the proposed RATCA method is utilized to track a threatening marine target continuously for imaging.

Some representative ISAR images from 11:55 to $12: 33$ are given in Figure 15. The conventional Range-Doppler method is utilized to obtain ISAR images of the target. The most important point is that the ISAR images are obtained continuously without any redetection. The proposed RATCA method ensures the validity of continuous imaging by tracking the target range and azimuth simultaneously within ISAR imaging processing. 


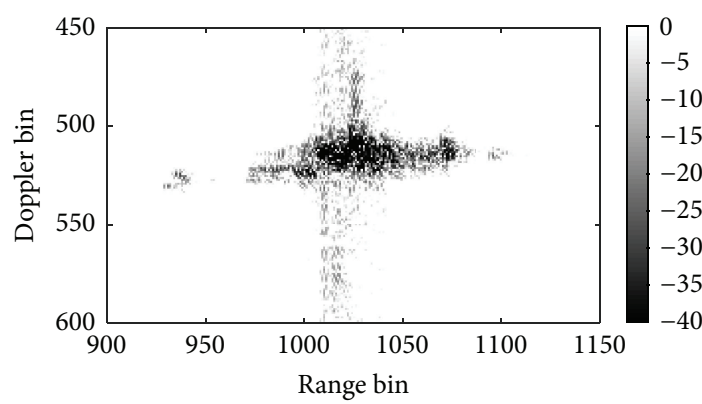

(a)

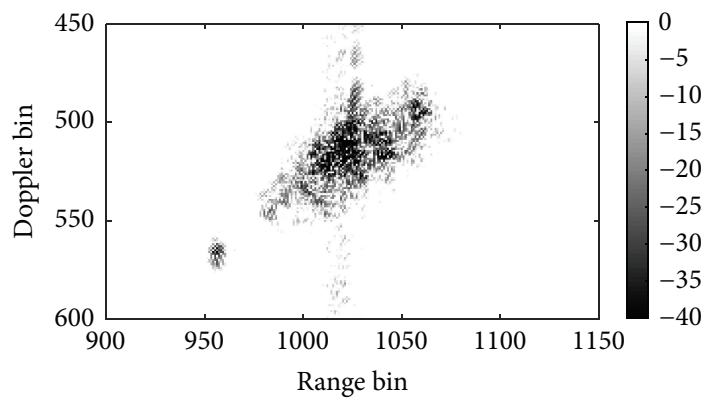

(c)

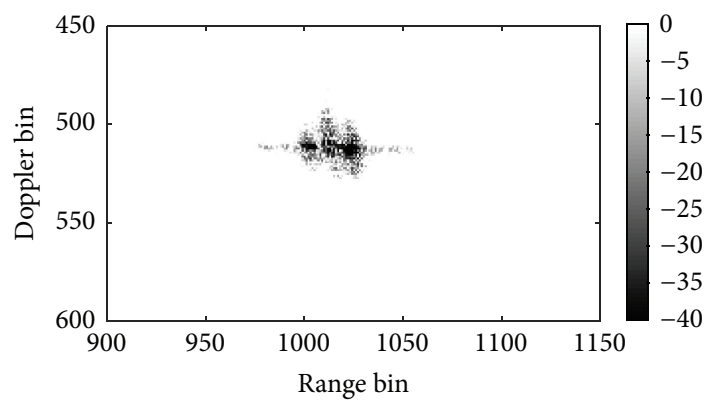

(e)

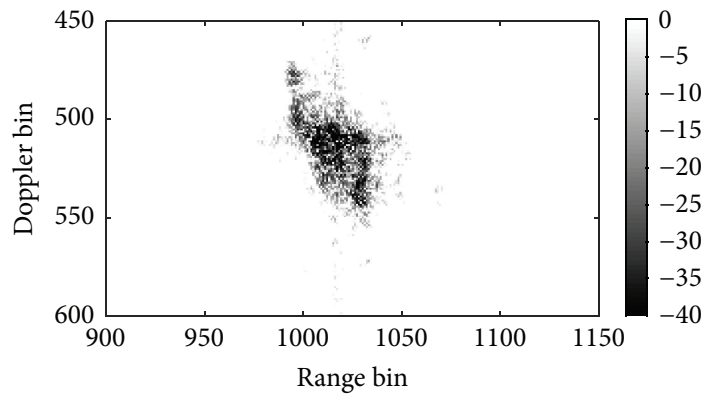

(g)

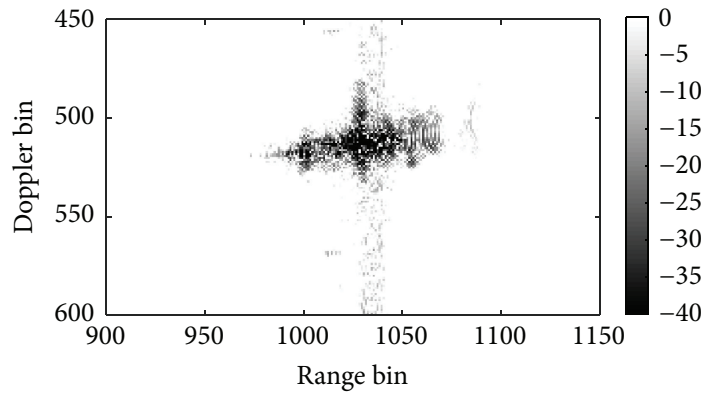

(i)

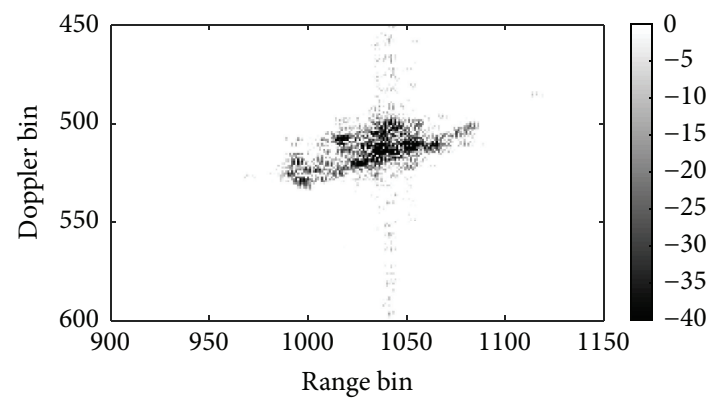

(b)

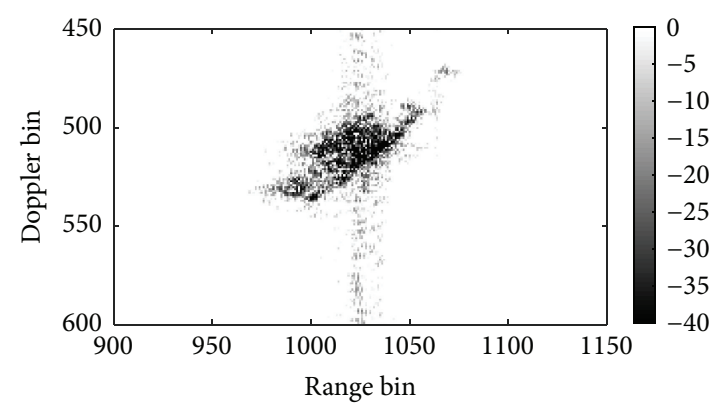

(d)

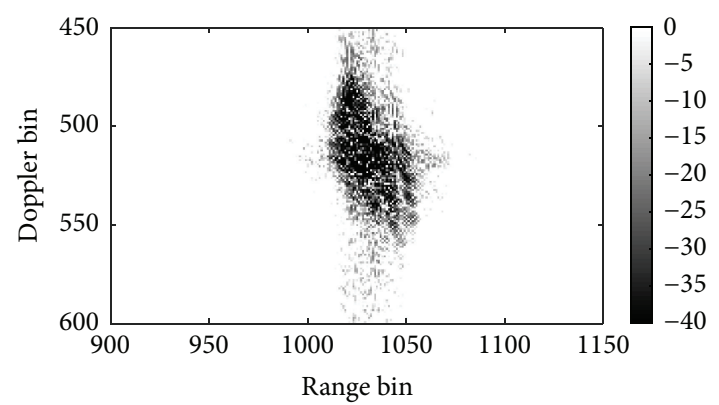

(f)

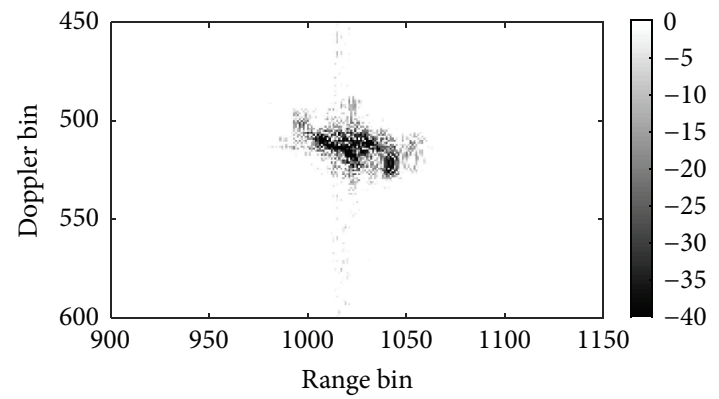

(h)

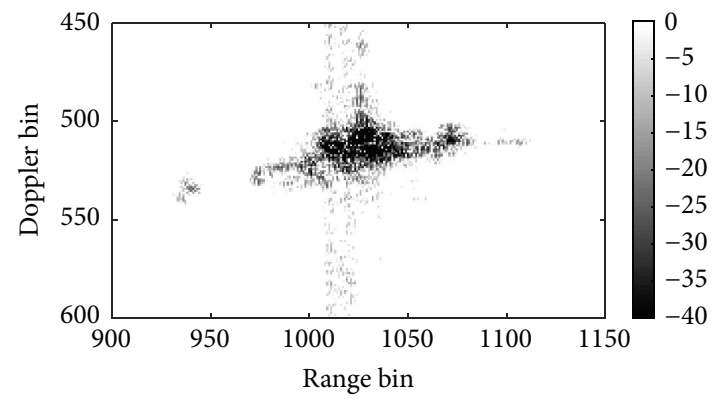

(j)

FIgURE 15: Continued. 


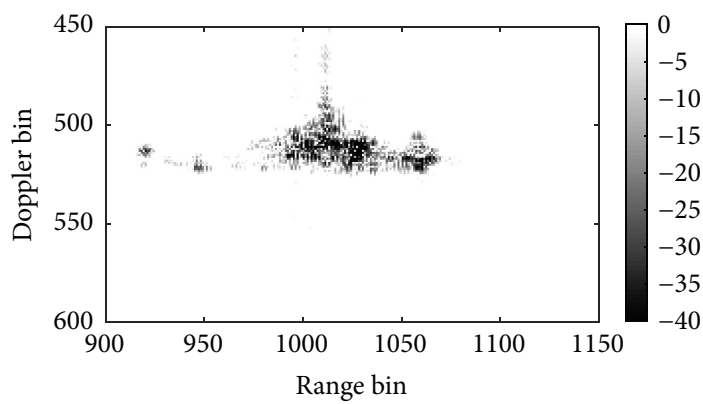

(k)

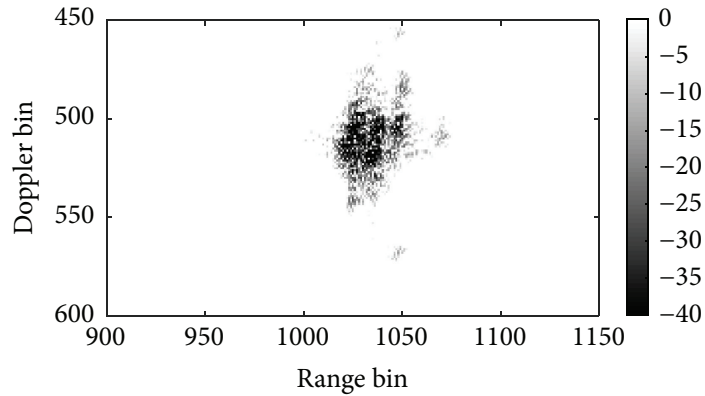

$(\mathrm{m})$

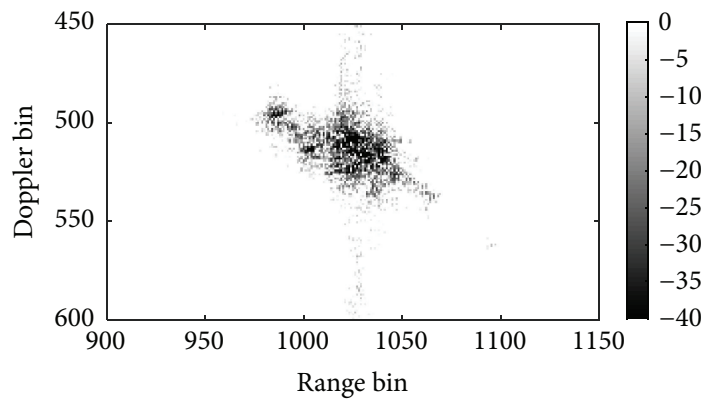

(o)

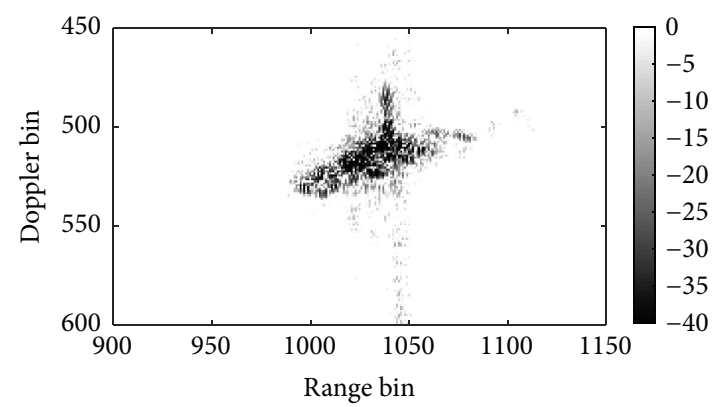

(1)

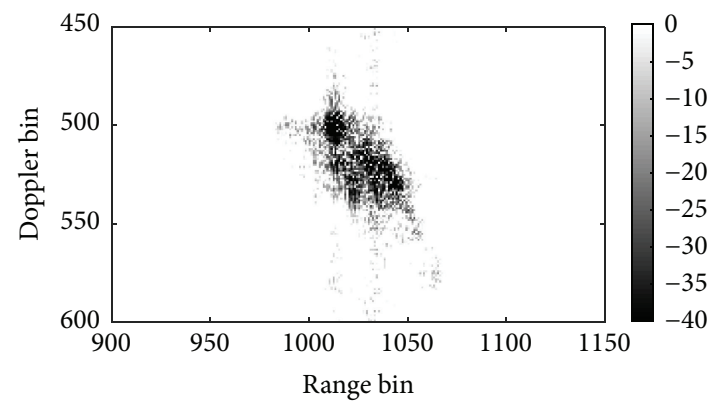

(n)

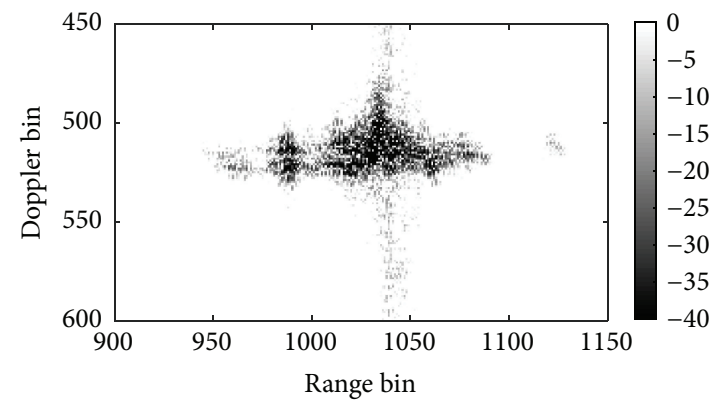

$(\mathrm{p})$

FIGURE 15: ISAR images of cooperative target. (a) Side-looking image at 11:55, (b) oblique-looking image at 11:58, (c) oblique-looking image at 12:01, (d) oblique-looking image at 12:04, (e) front-looking image at 12:06, (f) oblique-looking image at 12:08, (g) oblique-looking image at 12:11, (h) oblique-looking image at 12:14, (i) oblique-looking image at 12:17, (j) side-looking image at 12:20, (k) side-looking image at 12:22, (l) oblique-looking image at 12:23, (m) front-looking image at 12:26, (n) oblique-looking image at 12:27, (o) oblique-looking image at 12:29, and (p) side-looking image at 12:33.

The route of cooperative ship during the aforementioned time period consists of three parts in Figure 14: two straight lines and a turning point.

For convenience, we define several types of ISAR image attitude:

(1) Side-Looking Image. The ISAR image shows us a side view of the target when the true course of the target is 0 degrees relative to the antenna.

(2) Front-Looking Image. The ISAR image shows us a front view of the target when the true course of the target is 90 degrees relative to the antenna (meaning that the ship target only has tangential velocity toward the antenna with no radial motion).

(3) Oblique-Looking Image. The ISAR image shows us an oblique view between side-looking and front-looking images when the target has tangential and radial velocity relative to radar.

Figure 15(a) shows a side-looking image of the cooperative ship at 11:55 in which the true course of the target is approximately less than 10 degrees. The tilt of the ISAR image attitude, when the position of the target approaches the point marked by "12:06" where the heading changes, can be seen gradually from Figures 15(b)-15(d). Figure 15(e) illustrates the front-looking image of the target at 12:06, when the true course of the target is nearly 90 degrees toward the antenna. The cooperative target navigates straight away from radar between points marked by "12:06" and "12:23," where the true course increases from 90 to 180 degrees approximately; thus, the attitude of image tends to be side-looking again from Figures 15(f)-15(k). The rotation of navigation changes again around 12:23; thus, the tilt of ISAR image attitude 
can be shown from Figures 15(1)-15(o) (Figure 15(m) is the front-looking image). After turning back to the navigation direction, the cooperative ship target sails straight toward the platform where radar is located since 12:33 and only the sidelooking image of ISAR image could be illustrated, as shown in Figure 15(p).

\section{Conclusion}

In this paper, range and azimuth tracking in ISAR with wideband radar echoes is investigated. For the purpose of continuous imaging of threatening marine targets, the range and azimuth tracking (RAT) method is first presented for monopulse ISAR, which consists of two antenna feeds and could be regarded as the simplest phased array unit. However, the theoretical analysis and experimental results show that the RAT method is inappropriate for imaging in some extreme cases such as echo missing and jamming existence.

On the basis of centroid algorithm with forgotten factors, a modified approach for tracking range and azimuth simultaneously, namely, RATCA, is proposed to improve the tracking performance. The tracking performances with different forgotten factors are discussed versus velocity, acceleration, and SNR. Normally, the fluctuations of range and azimuth estimations with RATCA would be smoothed. More significantly, the modified method performs effectively when the target echo is missing occasionally. The range and azimuth are accurately estimated and the impacts of the erroneous estimations on the radar system are relieved. Compared with the RAT method, the RATCA method can be utilized to track a threatening marine target continuously for imaging, which is verified by the experimental results and numerous continuous ISAR images. The robustness and bias of the RATCA method are also theoretically analyzed. The method proposed in this paper is also applicable for phased array radar system, which could track multiple targets simultaneously.

\section{Competing Interests}

The authors declare that there are no competing interests regarding the publication of this paper.

\section{Acknowledgments}

This work was supported by a CASC-HIT United Technology Center key project (CASC-HIT11-1A01).

\section{References}

[1] C.-C. Chen and H. C. Andrews, "Target-motion-induced radar imaging," IEEE Transactions on Aerospace and Electronic Systems, vol. 16, no. 1, pp. 2-14, 1980.

[2] D. A. Ausherman, A. Kozma, J. L. Walker, H. M. Jones, and E. C. Poggio, "Developments in radar imaging," IEEE Transactions on Aerospace and Electronic Systems, vol. 20, no. 4, pp. 363-400, 1984.

[3] B. D. Steinberg, "Microwave imaging of aircraft," Proceedings of the IEEE, vol. 76, no. 12, pp. 1578-1592, 1988.
[4] S. K. Wong, G. Duff, and E. Riseborough, "Distortion in the inverse synthetic aperture radar (ISAR) images of a target with time-varying perturbed motion," IEE Proceedings: Radar, Sonar and Navigation, vol. 150, no. 4, pp. 221-227, 2003.

[5] V. C. Chen and W. J. Miceli, "Time-varying spectral analysis for radar imaging of manoeuvring targets," IEE ProceedingsRadar, Sonar and Navigation, vol. 145, no. 5, pp. 262-268, 1998.

[6] A. D. Lanterman, D. C. Munson Jr., and Y. Wu, "Wideangle radar imaging using time frequency distributions," IEE Proceedings: Radar, Sonar and Navigation, vol. 150, no. 4, pp. 203-211, 2003.

[7] Z. Bao, G. Wang, and L. Luo, "Inverse synthetic aperture radar imaging of maneuvering targets," Optical Engineering, vol. 37, no. 5, pp. 1582-1588, 1998.

[8] M. Xing, R. Wu, Y. Li, and Z. Bao, "New ISAR imaging algorithm based on modified Wigner-Ville distribution," IET Radar, Sonar and Navigation, vol. 3, no. 1, pp. 70-80, 2009.

[9] J. Li, C.-W. Qiu, L. Zhang, M. Xing, Z. Bao, and T.-S. Yeo, “Timefrequency imaging algorithm for highspeed spinning targets in two dimensions," IET Radar, Sonar and Navigation, vol. 4, no. 6, Article ID IRSNBX000004000006000806000001, pp. 806-817, 2010.

[10] D. L. Donoho, "Compressed sensing," IEEE Transactions on Information Theory, vol. 52, no. 4, pp. 1289-1306, 2006.

[11] E. J. Candès, J. Romberg, and T. Tao, "Robust uncertainty principles: exact signal reconstruction from highly incomplete frequency information," IEEE Transactions on Information Theory, vol. 52, no. 2, pp. 489-509, 2006.

[12] L. Zhang, M. Xing, C.-W. Qiu et al., "Resolution enhancement for inversed synthetic aperture radar imaging under low SNR via improved compressive sensing," IEEE Transactions on Geoscience and Remote Sensing, vol. 48, no. 10, pp. 3824-3838, 2010.

[13] G. Y. Delisle and H. Wu, "Moving target imaging and trajectory computation using ISAR," IEEE Transactions on Aerospace and Electronic Systems, vol. 30, no. 3, pp. 887-899, 1994.

[14] S. A. S. Werness, W. G. Carrara, L. S. Joyce, and D. B. Franczak, "Moving target imaging algorithm for SAR data," IEEE Transactions on Aerospace and Electronic Systems, vol. 26, no. 1, pp. 57-67, 1990.

[15] H. Wu and G. Y. Delisle, "Precision tracking algorithms for ISAR imaging," IEEE Transactions on Aerospace and Electronic Systems, vol. 32, no. 1, pp. 243-254, 1996.

[16] S. M. Sherman and D. K. Barton, Monopulse Principles and Techniques, Artech House, Norwood, Mass, USA, 1986.

[17] M. I. Skolnik, Radar Handbook, McGraw-Hill, New York, NY, USA, 2008.

[18] W. Zhou, J. Xie, Y. Yuan, and S. Li, "Angle measurement accuracy analysis of sum-difference amplitude-comparison monopulse in onshore or shipborne ISAR," in Proceedings of the 19th International Conference on Digital Signal Processing (DSP '14), pp. 531-535, IEEE, Hong Kong, August 2014. 


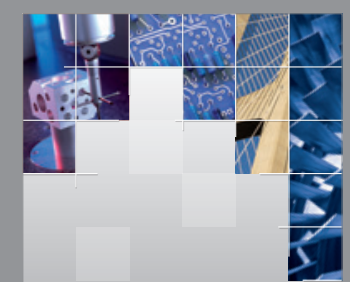

\section{Enfincering}
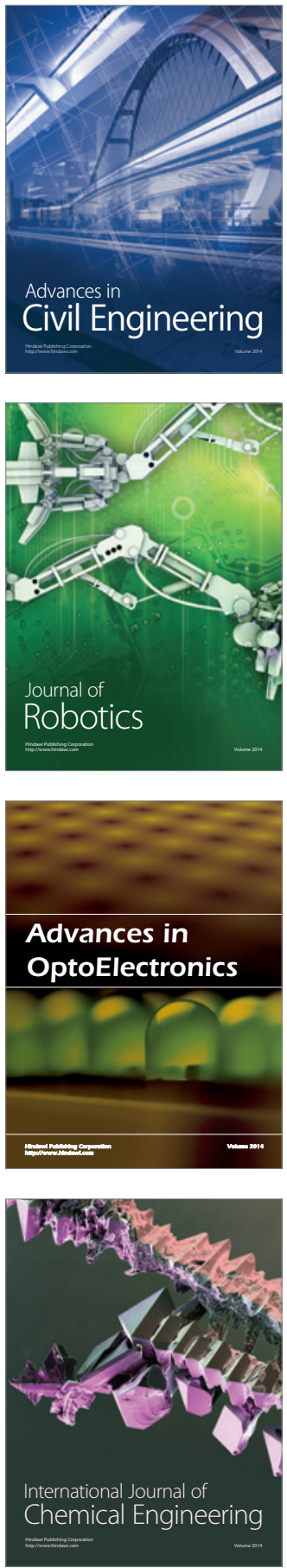

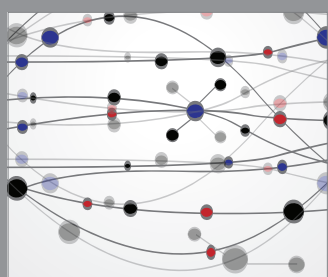

The Scientific World Journal

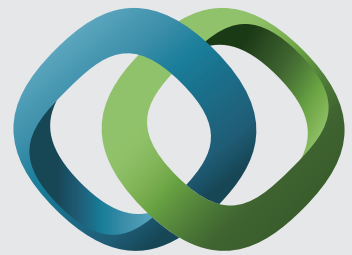

\section{Hindawi}

Submit your manuscripts at

http://www.hindawi.com
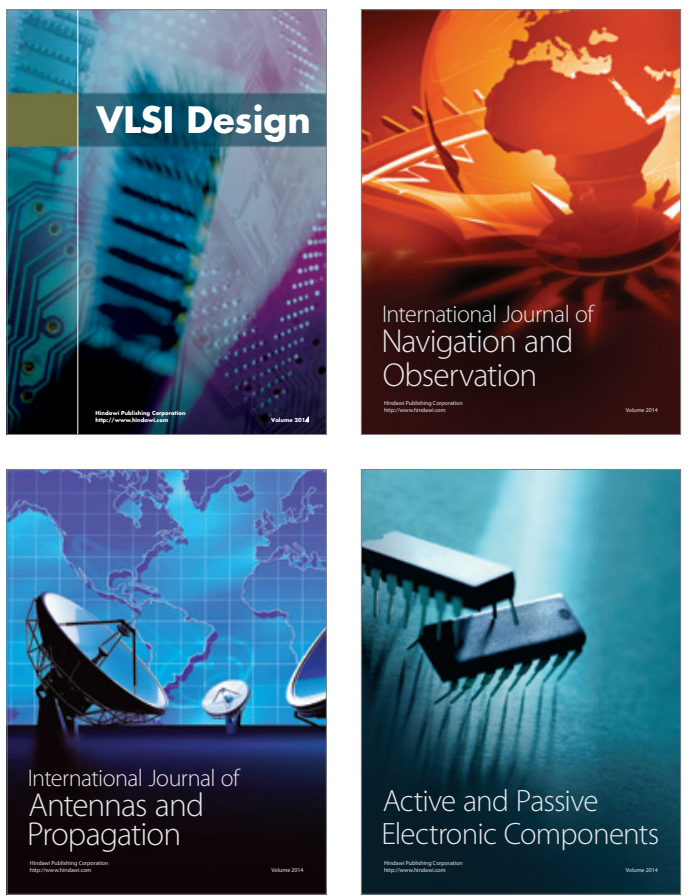
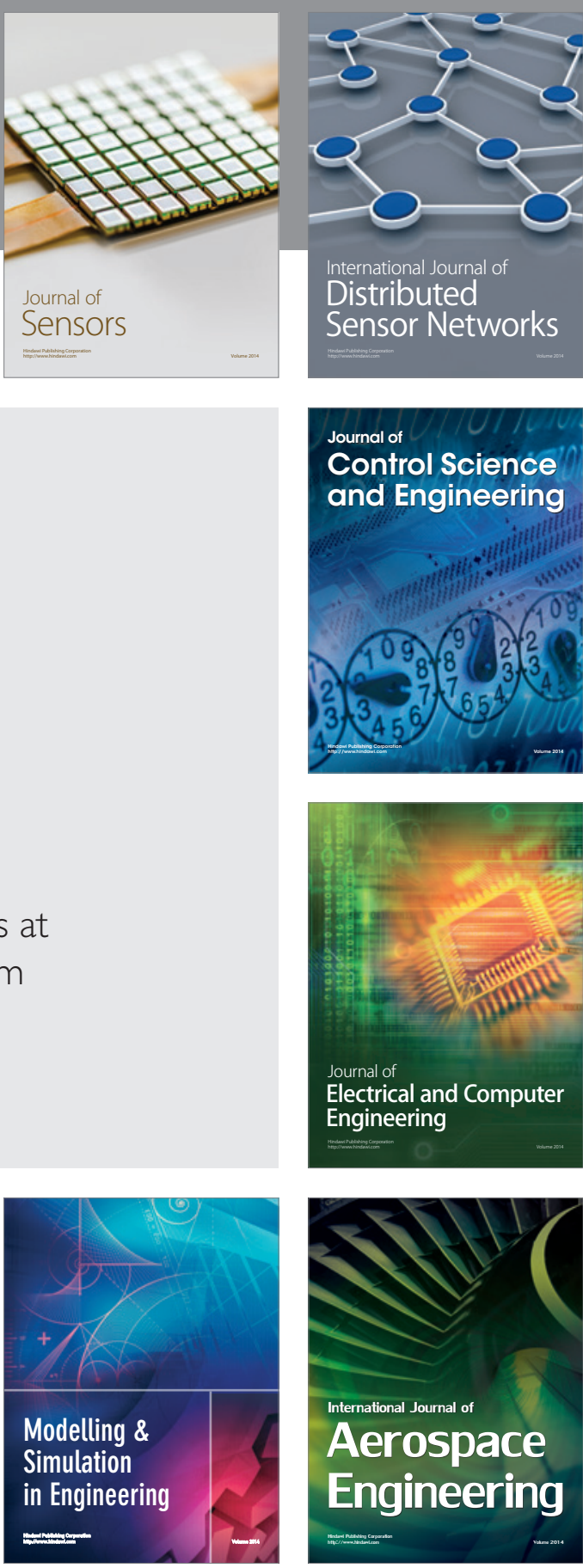

International Journal of

Distributed

Sensor Networks

Journal of

Control Science

and Engineering
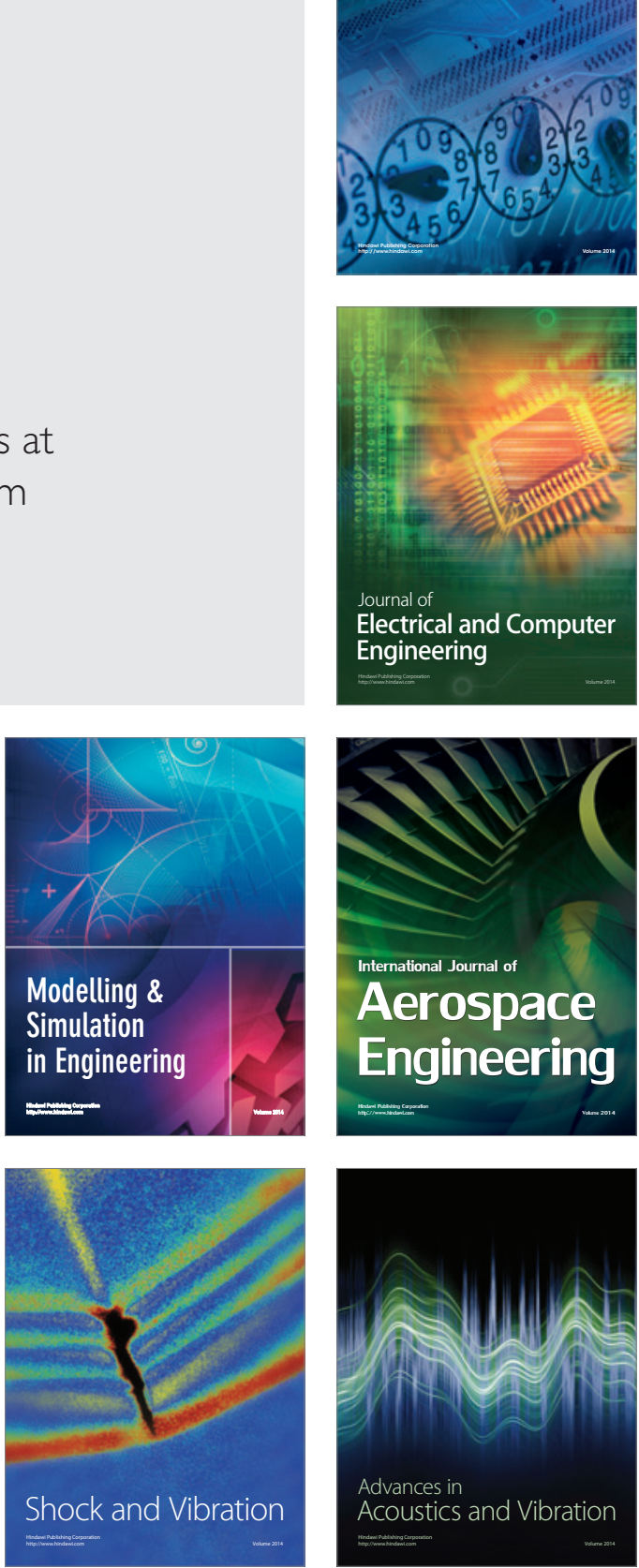